\title{
Counting Integer Points in Parametric Polytopes using Barvinok's Rational Functions
}

\author{
Sven Verdoolaege ${ }^{1}$, Rachid Seghir ${ }^{2}$, Kristof Beyls ${ }^{3}$, Vincent Loechner ${ }^{2}$, \\ Maurice Bruynooghe ${ }^{1}$ \\ 1 Dept. of Computer Science, Katholieke Universiteit Leuven, \\ e-mail: \{sven, maurice\}@cs. kuleuven.be \\ 2 ICPS-LSIIT UMR 7005, Université Louis Pasteur, Strasbourg, \\ e-mail: \{seghir, loechner\}@icps.u-strasbg.fr \\ 3 Dept. of Electronics and Information Systems, Ghent University, \\ e-mail: kristof.beyls@elis.UGent.be
}

\begin{abstract}
Many compiler optimization techniques depend on the ability to calculate the number of elements that satisfy certain conditions. If these conditions can be represented by linear constraints, then such problems are equivalent to counting the number of integer points in (possibly) parametric polytopes.

It is well known that the enumerator of such a set can be represented by an explicit function consisting of a set of quasi-polynomials each associated with a chamber in the parameter space. Previously, interpolation was used to obtain these quasi-polynomials, but this technique has several disadvantages. Its worstcase computation time for a single quasi-polynomial is exponential in the input size, even for fixed dimensions. The worst-case size of such a quasi-polynomial (measured in bits needed to represent the quasi-polynomial) is also exponential in the input size. Under certain conditions this technique even fails to produce a solution.

Our main contribution is a novel method for calculating the required quasipolynomials analytically. It extends an existing method, based on Barvinok's decomposition, for counting the number of integer points in a non-parametric polytope. Our technique always produces a solution and computes polynomially-sized enumerators in polynomial time (for fixed dimensions).
\end{abstract}

Key words Barvinok's decomposition - compiler analysis - Ehrhart quasi-polynomial - parametric polytope - polyhedral model - vector partition function signed unimodular decomposition

\section{Contents}

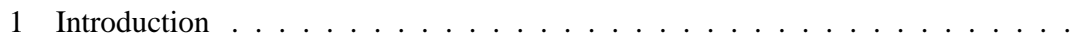




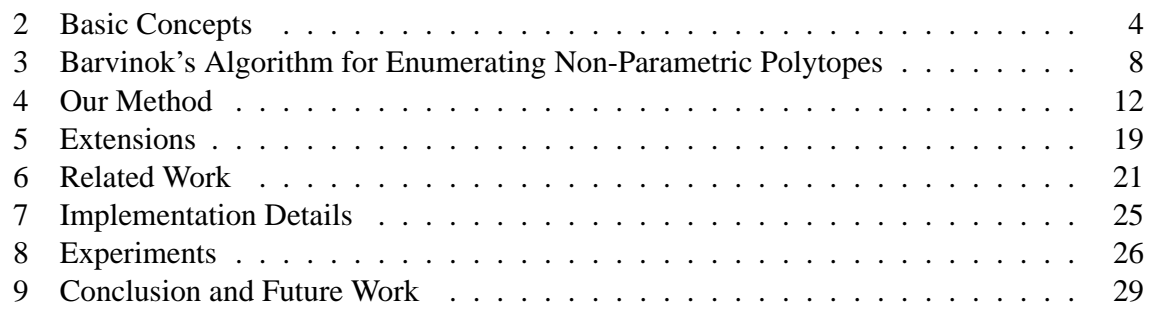

\section{Introduction}

In many program analyses and optimizations, questions starting with "how many" need to be answered, e.g.,

- How many memory locations are touched by a loop? [23]

- How many operations are performed by a loop? [35]

- How many cache lines are touched by a loop? [23]

- How many array elements are accessed between two points in time? [7]

- How many array elements are live at a given iteration $(i, j)$ ? [1,2,34,52]

- How many times is a statement executed before an iteration $(i, j)$ ? [48,25]

- How many parallel processing elements can be used when executing a loop on an FPGA? [6, 19,24,29]

- How many cache misses does a loop generate? [15, 13,27]

- How much memory is dynamically allocated by a piece of code? [11]

- How many different array elements are accessed before element $(x, y)$ is accessed? [38]

- How many bytes are transmitted between parallel tasks for a given parallel schedule of computation? [10,30,47]

Answering these questions is often one of the cornerstones of program transformations and optimizations, such as increasing parallelism [48], minimizing memory size [52,48], estimating worst case execution time [35], estimating energy consumption [33], increasing cache effectiveness [7], high-level transformations for DSP applications [25] and converting software loops into parallel hardware implementations [48,29]. Based on these program analyses, impressive program improvements have been reported, such as reducing memory size requirements in embedded systems from 262400 bytes to 257 bytes [34], increasing execution speed by a factor of 7 on DSP applications [25], and reducing execution time by a factor of 3.5 on a parallel distributed NUMA machine [30].

For many of the above analyses, the counting problem is formalized as counting the number of integer solutions to a system of linear inequalities, and the question becomes "How many integer points $\mathbf{x} \in \mathbb{Z}^{d}$ satisfy $A \mathbf{x} \geq \mathbf{b}$ ?", where $A$ is an integer matrix and $\mathbf{b}$ is an integer vector.

Furthermore, many analyses require the answer to be a function of some parameters $\mathbf{p}$, so that the number of integer points in the following set must be computed:

$$
P_{\mathbf{p}}=\left\{\mathbf{x} \in \mathbb{Q}^{d} \mid A \mathbf{x} \geq B \mathbf{p}+\mathbf{c}\right\},
$$




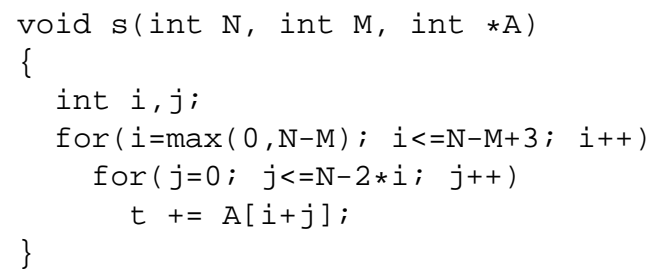

Fig. 1 Example loop code

where $\mathbf{p}$ is a vector of parameters, $A$ and $B$ are integer matrices, and $\mathbf{c}$ is an integer vector. $P_{\mathbf{p}}$ is called a parametric polytope, when the number of points satisfying $A \mathbf{x} \geq B \mathbf{p}+\mathbf{c}$ is finite for each value of $\mathbf{p}$. Clauss and Loechner [14] showed that the number of integer points in $P_{\mathbf{p}}$ can be represented by a set of quasi-polynomials, each valid on a different chamber. (These concepts will be explained in Section 2.2.) Consider, for example, the loop in Figure 1, and assume we wish to know how many times the statement $t+=A[i+j]$ executes, as a function of $N$ and $M$. The answer to this question can be computed by counting the number of integer points in

$$
P_{\left(\begin{array}{c}
N \\
M
\end{array}\right)}=\left\{\left(\begin{array}{l}
i \\
j
\end{array}\right) \in \mathbb{Q}^{2} \mid\left(\begin{array}{cc}
1 & 0 \\
1 & 0 \\
-1 & 0 \\
0 & 1 \\
-2 & -1
\end{array}\right)\left(\begin{array}{l}
i \\
j
\end{array}\right) \geq\left(\begin{array}{cc}
0 & 0 \\
1 & -1 \\
-1 & 1 \\
0 & 0 \\
-1 & 0
\end{array}\right)\left(\begin{array}{l}
N \\
M
\end{array}\right)+\left(\begin{array}{c}
0 \\
0 \\
-3 \\
0 \\
0
\end{array}\right)\right\} .
$$

The solution to this question is

$$
\# P_{\left(\begin{array}{c}
N \\
M
\end{array}\right)}= \begin{cases}-4 N+8 M-8 & \text { if } M \leq N \leq 2 M-7 \\
M N-2 N-M^{2}+6 M-8 & \text { if } N \leq M \leq N+3 \wedge N \leq 2 M-7 \\
\frac{N^{2}}{4}+\frac{3}{4} N+\frac{1}{2}\left\lfloor\frac{N}{2}\right\rfloor+1 & \text { if } 0 \leq N \leq M-1 \wedge 2 M \leq N+6 \\
\frac{N^{2}}{4}-M N-\frac{5}{4} N+M^{2}+2 M+\frac{1}{2}\left\lfloor\frac{N}{2}\right\rfloor+1 & \text { if } M \leq N \leq 2 M \leq N+6\end{cases}
$$

with $\lfloor x\rfloor$ the lower integer part of $x$. This solution contains 4 chambers, each with a different quasi-polynomial.

In some analyses, the need for parameters depends on the problem instance (e.g., in the presence of symbolic loop bounds), while in other techniques, e.g., $[7,11,13,23,25,35,38,48]$, the counting problem is intrinsically parametric. An example of an intrinsically parametric counting problem will be discussed in Example 10 in Section 5.2. It is also an example of a more general counting problem which requires some preprocessing to transform it into a system of constraints of the form (1). A number of the program analyses cited above $([1,2,7,10,13,15,29$, $30,33])$ require similar preprocessing. 
Recently, new techniques have been developed for similar counting problems, but they either do not support parameters, e.g., [9,41], or only to a very limited extent, e.g., LattE [18]. Clauss's method [14], on the other hand, does handle parameters but is worst-case exponential and the only known implementation even fails to produce a solution for some class of problems [48, 13].

In this paper, we present a novel method of enumerating parametric polytopes, which combines the decomposition into chambers of [14] and a parametric version of the counting algorithm in [18]. In contrast to previous methods, the resulting algorithm

- handles general parametric polytopes of the form (1),

- always produces an answer,

- computes quasi-polynomials that are only polynomially large in terms of the input size (for fixed dimensions), by using a representation for these quasipolynomials that differs from the one used in [14], and

- computes each of these quasi-polynomials in polynomial time, when the number of variables in the inequalities is fixed.

In the next section, the basic theoretical background is presented. Barvinok's algorithm for counting the number of integer points in a polytope is discussed in Section 3. In Section 4, the application of Barvinok's algorithm to parametric polytopes as in Equation (1) is introduced. Section 5 explains the preprocessing required to handle more general counting problems. A comparison with related work is performed in Section 6. Further details about the implementation of the algorithm are presented in Section 7. Section 8 evaluates our algorithm and compares it to Clauss's method, using a large set of systems of linear inequalities generated by several compiler analyses. Concluding remarks follow in Section 9. This paper is a revised version of [50].

\section{Basic Concepts}

In this section we first recall some basic polyhedral definitions. We further recall some theoretical results on polytope enumeration and introduce some new terminology.

\subsection{Basic Polyhedral Definitions}

Definition $1 A$ rational polyhedron $P \subset \mathbb{Q}^{d}$ is a set of rational d-dimensional vectors $\mathbf{x}$ defined by linear inequalities

$$
P=\left\{\mathbf{x} \in \mathbb{Q}^{d} \mid A \mathbf{x} \geq \mathbf{c}\right\}
$$

with $A \in \mathbb{Z}^{m \times d}$ and $\mathbf{c} \in \mathbb{Z}^{m}$.

Definition 2 The affine hull of a set $S \subset \mathbb{Q}^{d}$ is the set $\left\{\lambda_{1} x_{1}+\cdots+\lambda_{k} x_{k} \mid\right.$ $\left.\left\{x_{1}, \ldots x_{k}\right\} \subset S, \lambda_{i} \in \mathbb{Q}, \sum_{i} \lambda_{i}=1\right\}$. 
Definition 3 The dimension of a rational polyhedron $P \subset \mathbb{Q}^{d}$ is the dimension of its affine hull. Equivalently, it is equal to the dimension d of the ambient space $\mathbb{Q}^{d}$ minus the number of linearly independent (implicit) equalities in the system $A \mathbf{x} \geq \mathbf{c}$.

Definition $4 A$ face $F$ of a rational polyhedron $P(3)$ is the intersection of $P$ with $\left\{\mathbf{x} \in \mathbb{Q}^{d} \mid A^{\prime} \mathbf{x}=\mathbf{c}^{\prime}\right\}$, where $A^{\prime} \mathbf{x} \geq \mathbf{c}^{\prime}$ is a subsystem of $A \mathbf{x} \geq \mathbf{c}$. If $P$ has dimension $n$, then the $(n-1)$-dimensional faces are called facets. The 0-dimensional faces are called vertices.

By convention, the empty set $\emptyset$ is a (-1)-dimensional face of every polyhedron. Note that every vertex $\mathbf{v}$ of $P$ is an extremal point of $P$, i.e., $\mathbf{v}$ is a point that cannot be expressed as a convex combination of other points in $P$.

Definition 5 A rational polytope is a bounded rational polyhedron.

Definition 6 A rational parametric polytope $P_{\mathbf{p}}$ with $n$ parameters $\mathbf{p}$ is a set of rational d-dimensional vectors $\mathbf{x}$ defined by linear inequalities on $\mathbf{x}$ and $\mathbf{p}$

$$
P_{\mathbf{p}}=\left\{\mathbf{x} \in \mathbb{Q}^{d} \mid A \mathbf{x} \geq B \mathbf{p}+\mathbf{c}\right\}
$$

with $A \in \mathbb{Z}^{m \times d}, B \in \mathbb{Z}^{m \times n}$ and $\mathbf{c} \in \mathbb{Z}^{m}$, and such that for each fixed value $\mathbf{p}_{0}$ of $\mathbf{p}, P_{\mathbf{p}_{0}}$ defines a (possibly empty) rational polytope in $\mathbb{Q}^{d}$.

\subsection{Ehrhart Theory}

Definition 7 The enumerator $\mathcal{E}\left(P_{\mathbf{p}}\right)$ of a parametric polytope $P_{\mathbf{p}}$ is a function from the set of n-dimensional integer vectors $\mathbb{Z}^{n}$ to the set of natural numbers $\mathbb{N}$. The function value at $\mathbf{p}_{0}$, denoted $\mathcal{E}\left(P_{\mathbf{p}} ; \mathbf{p}_{0}\right)$, is the number of integer points in the polytope $P_{\mathbf{p}_{0}}$.

$$
\begin{aligned}
& \mathcal{E}\left(P_{\mathbf{p}}\right): \mathbb{Z}^{n} \rightarrow \mathbb{N} \\
& \mathbf{p}_{0} \mapsto \mathcal{E}\left(P_{\mathbf{p}} ; \mathbf{p}_{0}\right) \\
& \mathcal{E}\left(P_{\mathbf{p}} ; \mathbf{p}_{0}\right)=\#\left(\mathbb{Z}^{d} \cap\left\{\mathbf{x} \in \mathbb{Q}^{d} \mid A \mathbf{x} \geq B \mathbf{p}_{0}+\mathbf{c}\right\}\right)
\end{aligned}
$$

We first consider a special case of parametric polytopes $P_{\mathbf{p}}$ that can be written as the convex combination of a fixed set of parametric vertices, each linearly depending on the parameters, i.e.,

$$
P_{\mathbf{p}}=\left\{\mathbf{x} \in \mathbb{Q}^{d} \mid \mathbf{x}=\lambda V(\mathbf{p}), 0 \leq \lambda_{j}, \sum_{j} \lambda_{j}=1\right\},
$$

with the columns $V_{j}(\mathbf{p})$ of $V(\mathbf{p})$ the vertices of $P_{\mathbf{p}}$, i.e., $V_{j}(\mathbf{p})=G_{j} \mathbf{p}+\mathbf{h}_{j}$ for some $G_{j} \in \mathbb{Q}^{d \times n}$ and $\mathbf{h}_{j} \in \mathbb{Q}^{d}$. Ehrhart [21] showed that the enumerator of such a set can be represented as a quasi-polynomial. Before we can define a quasi-polynomial, we need the concept of a periodic number. 
Definition 8 ([14]) A rational $n$-periodic number $U(\mathbf{p})$ is a function $\mathbb{Z}^{n} \rightarrow \mathbb{Q}$, such that there exist a period $\mathbf{q}=\left(q_{1}, \ldots, q_{n}\right) \in \mathbb{N}^{n}$ such that $U(\mathbf{p})=U\left(\mathbf{p}^{\prime}\right)$ whenever $p_{i} \equiv p_{i}^{\prime} \bmod q_{i}$, for $1 \leq i \leq n$.

Definition 9 A quasi-polynomial of degree $d$ in $n$ variables $\mathbf{p}$ is a polynomial expression of degree d in $\mathbf{p}$ over the rational n-periodic numbers, i.e.,

$$
\sum_{\mathbf{i} \in I} U_{\mathbf{i}}(\mathbf{p}) \mathbf{p}^{\mathbf{i}}
$$

with $I \subset \mathbb{N}^{d}$, $U_{\mathbf{i}}$ periodic numbers and $\mathbf{p}^{\mathbf{i}}=p_{1}^{i_{1}} p_{2}^{i_{2}} \cdots p_{d}^{i_{d}}$. The $\mathbf{p e r i o d}$ of a quasipolynomial is the componentwise least common multiple $(\mathrm{lcm})$ of the periods of its coefficients $U_{\mathbf{i}}$.

In other words, the coefficients of a quasi-polynomial depend periodically on the variables. This definition is a natural generalization of the "classical" quasipolynomials in a single variable [45]. Such univariate quasi-polynomials can also be seen as a list of $q$ polynomials, with $q$ the one-dimensional period of the quasipolynomial. Similarly, a multivariate quasi-polynomial can be considered as an $n$-dimensional array of polynomials.

Since many of these polynomials share coefficients, the representation suggested by Definition 9 and used in [14] seems more appropriate than the use of a list or an array. The periodic numbers can be represented by an $n$-dimensional lookup-table $U_{\mathbf{p}}$ such that $U(\mathbf{p})=U_{\mathbf{p}}\left[p_{1} \bmod q_{1}, \ldots, p_{n} \bmod q_{n}\right]$. Such lookuptables are typically written as a list or matrix of values enclosed in square brackets. E.g., $U_{N}=[1,3 / 4]_{N}$ is a 1-periodic number with period $q_{1}=2 ; U_{N}=1$ if $N \bmod 2 \equiv 0$ and $U_{N}=3 / 4$ if $N \bmod 2 \equiv 1$.

Lemma 1 ([14]) Given a parametric polytope that can be written as a convex combination of a set of parametric vertices $\mathbf{v}_{j}(\mathbf{p})$ as in (5), the period in dimension $i$ of the periodic numbers that appear in the enumerator of the polytope is a divisor of the lcm of the denominators that appear in the coefficients of $p_{i}$ in the affine expressions that define the vertices.

Since the periods $q_{i}$ are bounded only by the value of the coefficients in the input, they can be exponential in terms of the input size and so the worst-case size of a quasi-polynomial in this representation is exponential in the input size. Recall that the input size is the number of bits needed to represent the input polytope [43]. The representation as an $n$-dimensional array obviously shares this worstcase exponential size property. We will therefore prefer a different representation.

Definition 10 A step-polynomial of degree $d$ in $\mathbf{p}$ is a degree-d polynomial expression in the lower integer parts (floors) of rational affine expressions in $\mathbf{p}$.

Lemma 2 A step-polynomial of degree din $\mathbf{p}$ is a quasi-polynomial of degree din p.

To see that such a step-polynomial is indeed a quasi-polynomial in $\mathbf{p}$, rewrite each lower integer part as $\lfloor f(\mathbf{p})\rfloor=f(\mathbf{p})-\{f(\mathbf{p})\}$, with $\{x\}$ the fractional part 
of $x$ and $f(\mathbf{p})=a_{0}+\sum_{i} a_{i} p_{i}$. The fractional part $\{f(\mathbf{p})\}$ is a periodic number with as period the denominators of $a_{i}$. If the degree of such a step-polynomial is fixed and if the number of different rational affine expressions is polynomial in the input size, then so is the total size of this step-polynomial.

Example 1 Consider the step-polynomial $\frac{1}{4}\lfloor N\rfloor^{2}+\frac{3}{4}\lfloor N\rfloor+\frac{1}{2}\left\lfloor\frac{N}{2}\right\rfloor+1$. This steppolynomial can be rewritten as

$$
\frac{1}{4}(N-\{N\})^{2}+\frac{3}{4}(N-\{N\})+\frac{1}{2}\left(\frac{N}{2}-\left\{\frac{N}{2}\right\}\right)+1=\frac{N^{2}}{4}+N-\frac{1}{2}\left\{\frac{N}{2}\right\}+1,
$$

where $\{N\}=0$ since we are only interested in evaluation at integer values. Clearly, $1-\frac{1}{2}\left\{\frac{N}{2}\right\}$ is also a periodic number and equals $[1,3 / 4]_{N}$.

Loechner $[36,14]$ showed that the parameter space of a general parametric polytope $P_{\mathbf{p}}(1)$ can be divided into a set of chambers such that each chamber $P_{\mathbf{p}}$ has a fixed set of parametric vertices that are affine combinations of the parameters ${ }^{1}$ In each chamber, the parametric polytope can therefore be written as (5). Section 4.1 reviews the chamber decomposition algorithm and provides a complexity analysis. Using this decomposition, Clauss and Loechner [14] proved the following proposition.

Proposition 1 The enumerator of a d-dimensional parametric polytope $P_{\mathbf{p}}$ is a quasi-polynomial of degree $d$ in $\mathbf{p}$ on each of a set of chambers that form a subdivision of the parameter space. The period of the quasi-polynomial in a given chamber divides the denominators that appear in the vertices defined on that chamber (componentwise).

Note that this proposition generalizes the result of Sturmfels [46], who considered the enumerators of a special case of parametric polytopes, called vector partition functions. Likewise, our chambers are a generalization of the chambers introduced in that paper. In particular, the chambers of a vector partition function are always cones.

Example 2 In the chamber $0 \leq N \leq M-1 \wedge 2 M \leq N+6$, the parametric polytope in Equation (2) has vertices $(0,0),(0, N)$ and $\left(\frac{N}{2}, 0\right)$. Based on the denominators, the period is 2 in the dimension of parameter $N$ and 1 in the dimension of parameter $M$. Therefore, the quasi-polynomial for this chamber has the following form

$$
[a, b]_{N} N^{2}+[c, d]_{N} N M+[e, f]_{N} M^{2}+[g, h]_{N} M+[i, j]_{N} N+[k, l]_{N},
$$

where unknowns $a, \ldots, l$ are rational numbers. After finding the correct values for $a, \ldots, l$, the solution can be written as:

$$
\frac{1}{4} N^{2}+N+\left[1, \frac{3}{4}\right]_{N}
$$

which equals the step-polynomial $\frac{N^{2}}{4}+\frac{3 N}{4}+\frac{1}{2}\left\lfloor\frac{N}{2}\right\rfloor+1$.

\footnotetext{
${ }^{1}$ Clauss and Loechner [14] uses the term "validity domain" instead of chamber.
} 


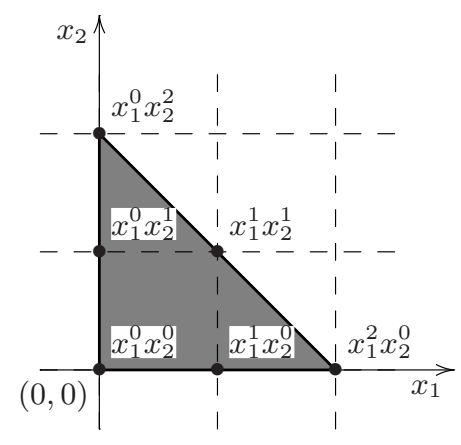

Fig. 2 Barvinok Example. For each integer point $(i, j)$ in the polytope $T$, there is a term $x_{1}^{i} x_{2}^{j}$ in the generating function $f(T ; \mathbf{x})$.

\section{Barvinok's Algorithm for Enumerating Non-Parametric Polytopes}

In this section we review Barvinok's method for computing the number of integer points in a polytope. This method consists of two steps. First, what is known as the generating function of a polytope is computed. In the second step, this generating function is evaluated to obtain the number of integer points in the polytope. Whenever we refer to Barvinok's algorithm we mean the algorithm as outlined by Barvinok [3] and further refined by Yoshida et al. [18,51]. Without loss of generality (see Section 7), we assume throughout that the polytope $P \subset \mathbb{Q}^{d}$ is fulldimensional, i.e., that the dimension of $P$ is $d$ as well. Results cited from other sources have been adapted accordingly.

\subsection{The Generating Function of a Polytope}

The basic idea behind Barvinok's algorithm is to consider the generating function of the integer points in a polytope $P$. This generating function is a formal power series with a term for each integer point in $P$, i.e.,

$$
f(P ; \mathbf{x})=\sum_{\boldsymbol{\alpha} \in P \cap \mathbb{Z}^{d}} \mathbf{x}^{\boldsymbol{\alpha}},
$$

with $\mathrm{x}^{\alpha}=x_{1}^{\alpha_{1}} x_{2}^{\alpha_{2}} \cdots x_{d}^{\alpha_{d}}$. Evaluating this function at $\mathbf{x}=\mathbf{1}$ yields the number of terms, which equals the desired number of points. The generating function is obviously not constructed by enumerating all the integer points in $P$, but rather as a signed sum of rational functions that can be derived from the description of $P$.

Example 3 Consider the polytope $T=\left\{\mathbf{x} \mid x_{1} \geq 0 \wedge x_{2} \geq 0 \wedge x_{1}+x_{2} \leq 2\right\}$ shown in Figure 2. This is a slightly modified version of the polytope in [18, Example 7]. The integer points in $T$ are $(0,0),(1,0),(2,0),(0,1),(1,1)$, and $(2,0)$. The generating function of $T$ is therefore $f(T ; \mathbf{x})=1+x_{1}+x_{1}^{2}+x_{2}+x_{1} x_{2}+x_{2}^{2}$. Barvinok's algorithm, however, will produce this function in a different form which does 
not require the computation of all integer points in the polytope. In particular, it will compute $f(T ; \mathbf{x})$ as the following short sum of rational functions:

$$
f(T ; \mathbf{x})=\frac{x_{1}^{2}}{\left(1-x_{1}^{-1}\right)\left(1-x_{1}^{-1} x_{2}\right)}+\frac{x_{2}^{2}}{\left(1-x_{2}^{-1}\right)\left(1-x_{1} x_{2}^{-1}\right)}+\frac{1}{\left(1-x_{1}\right)\left(1-x_{2}\right)} .
$$

Before describing how Barvinok constructs the generating function, we first introduce some more definitions.

Definition 11 A cone with generators $\mathbf{u}_{1}, \mathbf{u}_{2}, \ldots, \mathbf{u}_{k} \in \mathbb{Z}^{d}$ is a set of the form $\left\{\sum_{i} \lambda_{i} \mathbf{u}_{i} \mid \lambda_{i} \geq 0\right\}$. It is called unimodular if its generators form a basis of $\mathbb{Z}^{d}$.

Definition 12 Given a polyhedron $P$ and one of its vertices $\mathbf{v}$, the vertex cone cone $(P, \mathbf{v})$ of $P$ at $\mathbf{v}$ is the region of $\mathbb{Q}^{d}$ bounded by the constraints of $P$ that $\mathbf{v}$ saturates ${ }^{2}$ I.e., it is the region bounded by the constraints $\langle\mathbf{a}, \mathbf{x}\rangle \geq b$ with $\langle\mathbf{a}, \mathbf{v}\rangle=$ $b$, where $\langle.,$.$\rangle is the standard inner product.$

The polytope $T$ from Example 3 has vertices $(0,2),(2,0)$, and $(0,0)$. The vertex cones that correspond to these vertices are $\left\{\mathbf{x} \mid x_{2} \geq 0 \wedge x_{1}+x_{2} \leq 2\right\}$, $\left\{\mathbf{x} \mid x_{1} \geq 0 \wedge x_{1}+x_{2} \leq 2\right\}$, and $\left\{\mathbf{x} \mid x_{1} \geq 0 \wedge x_{2} \geq 0\right\}$ respectively. Note that according to Definition 11, a vertex cone $\operatorname{cone}\left(P, \mathbf{v}_{i}\right)$ is not a cone itself, but the sum of $\mathbf{v}_{i}$ and some cone $K$. According to Brion's theorem [12], the generating function of $P$ is equal to the sum of the generating functions of its vertex cones.

In the simplest case, all vertices are integer and all vertex cones are translates of unimodular cones. The generating function of a unimodular cone $K_{i}$ is simply

$$
f\left(K_{i} ; \mathbf{x}\right)=\prod_{j=1}^{k} \frac{1}{\left(1-\mathbf{x}^{\mathbf{u}_{j}^{i}}\right)}
$$

with $\mathbf{u}_{j}^{i}$ the generators of $K_{i}$. (This is a natural generalization of the geometric series to multiple dimensions; see, e.g., [3].) The generating function of the vertex cone is obtained by translating the generating function of the corresponding unimodular cone $K_{i}$ to the vertex $\mathbf{v}_{i}$. If $\mathbf{v}$ is integer, then we simply need to add $\mathbf{v}$ to all the exponents in the generating function, which corresponds to a multiplication by $\mathrm{x}^{\mathbf{v}}$. Applied to the polytope $T$ from Example 3, the generators for the respective vertex cones are $(1,0),(0,1),(-1,0)(-1,1)$, and $(0,-1),(1,-1)$. Translation with, respectively, $x_{1}^{2}, x_{2}^{2}$, and $x_{1}^{0} x_{2}^{0}$ followed by summation yields the generating function shown in Equation (7).

If some of the (translated) vertex cones are not unimodular, then the computation is more complicated. Barvinok proposed to decompose such a cone into a signed "sum" of unimodular cones $\left\{\left(\epsilon_{i}, K_{i}\right)\right\}=\mathcal{B}(K)$, with $\epsilon_{i} \in\{-1,1\}$, the sign corresponding to unimodular cone $K_{i}$. Here, "sum" can be interpreted to mean that the generating function of $K$ is the signed sum of the generating functions of the unimodular cones (8). A key feature of this signed decomposition

\footnotetext{
${ }^{2}$ A vertex cone is also known as a supporting cone.
} 
is that the number of elements in $\mathcal{B}(K)$ as well as the total computation time is polynomial in the input size (for fixed dimensions).

To obtain the final generating function, the generating functions corresponding to the unimodular cones $K_{i}$ need to be translated to the vertex $\mathbf{v}$. As explained above, if $\mathbf{v}$ is integer, then we simply need to add $\mathbf{v}$ to all the exponents in the generating function. If $\mathbf{v}$ is not integer, however, then we need to find an integer point $\mathbf{v}^{\prime}=E\left(\mathbf{v}, K_{i}\right)$ such that $\left(K_{i}+\mathbf{v}^{\prime}\right) \cap \mathbb{Z}^{d}=\left(K_{i}+\mathbf{v}\right) \cap \mathbb{Z}^{d}$. Since $K_{i}$ is unimodular, this point exists and is uniquely defined as the integer linear combination of the generators of $K_{i}$ with the smallest coefficients that lies inside $K_{i}+\mathbf{v}$ [18]. I.e.,

$$
E\left(\mathbf{v}, K_{i}\right)=\sum_{j}\left\lceil\lambda_{j}\right\rceil \mathbf{u}_{j}^{i}
$$

where $\boldsymbol{\lambda}$ is the rational solution to $\mathbf{v}=\sum_{j} \lambda_{j} \mathbf{u}_{j}^{i}$ and $\lceil$.$\rceil is the upper integer part.$ Note that if $\mathbf{v}$ is integer, then $E\left(\mathbf{v}, K_{i}\right)=\mathbf{v}$. The final generating function is then

$$
f(P ; \mathbf{x})=\sum_{\mathbf{v} \in \mathcal{V}(P)} \sum_{i=1}^{\left|\mathcal{B}\left(K_{\mathbf{v}}\right)\right|} \epsilon_{i} \frac{\mathbf{x}^{E\left(\mathbf{v}, K_{i}\right)}}{\prod_{j=1}^{d}\left(1-\mathbf{x}^{\mathbf{u}_{j}^{i}}\right)},
$$

with $\mathcal{V}(P)$ the parametric vertices of $P$. For a proof of the correctness of this formula, see [3].

Example 4 Figure 3 shows a polytope $P$ (shaded area) and its vertex cone (thick lines) at vertex $\mathbf{v}_{1}$. A possible signed unimodular decomposition for $\operatorname{cone}\left(P, \mathbf{v}_{1}\right)-$ $\mathbf{v}_{1}$ is the pair $\left\{\left(1, K_{1}\right),\left(1, K_{2}\right)\right\}$. ${ }^{3}$ Let $\mathbf{v}_{1}^{\prime}=E\left(\mathbf{v}_{1}, K_{1}\right)$ and $\mathbf{v}_{1}^{\prime \prime}=E\left(\mathbf{v}_{1}, K_{2}\right)$. In the figure, $\mathbf{v}_{1}^{\prime}=(1,0)$ and $\mathbf{v}_{1}^{\prime \prime}=(1,1)$. Since both signs are positive, we have

$$
f\left(\operatorname{cone}\left(P, \mathbf{v}_{1}\right) ; \mathbf{x}\right)=f\left(\mathbf{v}_{1}^{\prime}+K_{1} ; \mathbf{x}\right)+f\left(\mathbf{v}_{1}^{\prime \prime}+K_{2} ; \mathbf{x}\right) .
$$

The integer points in $\mathbf{v}_{1}^{\prime \prime}+K_{2}$ are marked by $\boxplus$, whereas those in $\mathbf{v}_{1}^{\prime}+K_{1}$ are marked by $\oplus$.

\subsection{Evaluation of the Generating Function}

The previous section explained how to compute the generating function of a polytope. Note that each term of this generating function (10) has a pole at $\mathbf{x}=1$. The total sum, however, is clearly analytic at $\mathbf{x}=1$. The coefficients of the negative powers in the Laurent series expansion of the terms at $x=1$ therefore cancel each other and the value at $\mathbf{x}=\mathbf{1}$ is simply the sum of the coefficients of the constant term in the Laurent series of each of the terms in (10). This computation can be simplified by first transforming the multivariate rational functions to univariate rational functions. Following Yoshida et al. [18,51], we choose an integer vector $\boldsymbol{\mu}$ not orthogonal to any generator and perform the variable substitution

\footnotetext{
${ }^{3}$ Note that this is not the decomposition that our implementation would produce, since, like Yoshida [18], we perform the decomposition on the dual cone.
} 


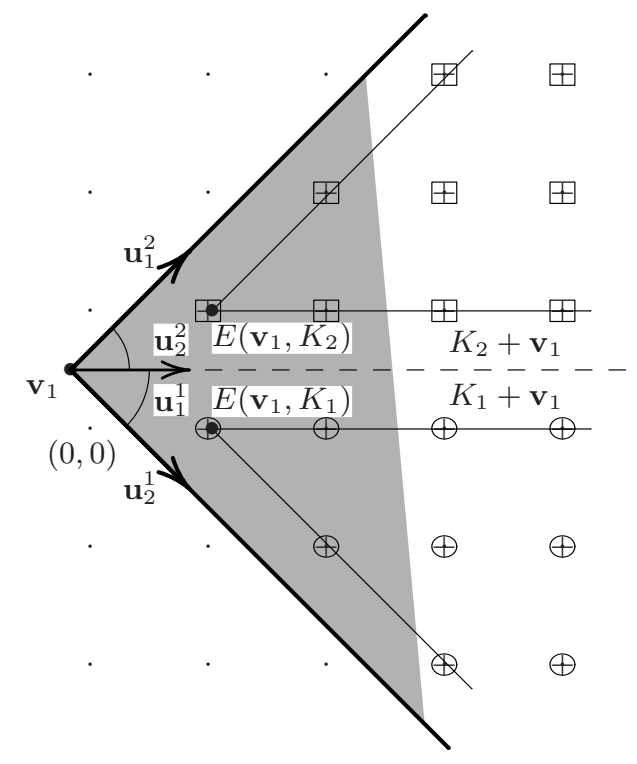

Fig. 3 Barvinok's decomposition of cone $\left(P, \mathbf{v}_{1}\right)$. The vertex cone (thick lines) at vertex $\mathbf{v}_{1}$ translated to the origin is decomposed into two unimodular cones $K_{1}$ with generators $\left(\mathbf{u}_{1}^{1}, \mathbf{u}_{2}^{1}\right)$ and $K_{2}$ with generators $\left(\mathbf{u}_{1}^{2}, \mathbf{u}_{2}^{2}\right) . E\left(\mathbf{v}_{1}, K_{1}\right)$ and $E\left(\mathbf{v}_{1}, K_{2}\right)$ are the integer points closest to $\mathbf{v}_{1}$ in cones $K_{1}+\mathbf{v}_{1}$ and $K_{2}+\mathbf{v}_{1}$.

$x_{i}=(s+1)^{\mu_{i}}$. Each (multivariate) term in (10) can then be written as the univariate

$$
\epsilon_{i}^{\prime} \frac{N(s)}{D^{\prime}(s)}=\epsilon_{i}^{\prime} \frac{(s+1)^{\left\langle\boldsymbol{\mu}, E\left(\mathbf{v}, K_{i}\right)\right\rangle-c}}{s^{d} D(s)},
$$

with $D(s)$ a non-zero polynomial with integer coefficients, independent of $\mathbf{v}$, and $c$ the sum of the negative $\left\langle\boldsymbol{\mu}, \mathbf{u}_{j}^{i}\right\rangle$ s. Evaluating (10) at $\mathbf{x}=\mathbf{1}$ is equivalent to summing the coefficients of $s^{0}$ in the Laurent series of each of the terms (11). This in turn can be accomplished by computing the coefficient of $s^{d}$ in the Taylor expansion of $N(s) / D(s)$. Note that this computation only requires the first $(d+1)$ coefficients of $N(s)$. The (signed) sum of the coefficients of $s^{d}$ in each of the terms then yields the desired number of points in the polytope.

Example 5 Consider once more the polytope $T$ from Example 3. Since each of its vertex cones is unimodular, the generating function $f(T ; \mathbf{x})$ has exactly one term for each vertex. To evaluate $f(T ; \mathbf{x})$ at $\mathbf{1}$ one can take $\boldsymbol{\mu}=(1,-1)$ since it is not orthogonal to any generator. Substituting $x_{1}=(s+1)^{\mu_{1}}$ and $x_{2}=(s+1)^{\mu_{2}}$ in $f(T ; \mathbf{x})(7)$, we obtain:

$$
\begin{aligned}
f(T ; s)=\frac{(s+1)^{2}}{\left(1-(s+1)^{-1}\right)\left(1-(s+1)^{-2}\right)}+ \\
\quad \frac{(s+1)^{-2}}{(1-(s+1))\left(1-(s+1)^{2}\right)}+\frac{1}{(1-(s+1))\left(1-(s+1)^{-1}\right)} .
\end{aligned}
$$


In order to obtain only positive powers in the denominators, we multiply the numerator and the denominator of each term by $(s+1)^{-c}$, with $c$ the sum of the negative powers in the denominator. Here, $c$ equals $-3,0$ and -1 respectively. This returns $f(T ; s)$ in the form:

$$
\begin{aligned}
f(T ; s)= & \frac{(s+1)^{5}}{(1-(s+1))\left(1-(s+1)^{2}\right)}+ \\
& \frac{(s+1)^{-2}}{(1-(s+1))\left(1-(s+1)^{2}\right)}-\frac{(s+1)}{(1-(s+1))(1-(s+1))}
\end{aligned}
$$

or

$$
f(T ; s)=\frac{(s+1)^{5}}{s^{2}(s+2)}+\frac{(s+1)^{-2}}{s^{2}(s+2)}-\frac{(s+1)}{s^{2}} .
$$

Using the (truncated) expansions

$$
\frac{1}{s+2} \equiv \frac{1}{2}\left(1-\frac{1}{2} s+\frac{1}{4} s^{2}\right) \quad \bmod s^{3}
$$

and

$$
(s+1)^{n} \equiv 1+n s+\frac{n(n-1)}{2} s^{2} \quad \bmod s^{3} \quad \text { for } n \in\{-2,5\}
$$

we obtain

$s^{2} f(T ; s) \equiv\left[\frac{1}{2}+\frac{9}{4} s+\frac{31}{8} s^{2}\right]+\left[\frac{1}{2}-\frac{5}{4} s+\frac{17}{8} s^{2}\right]-\left[1+s+0 s^{2}\right] \bmod s^{3}$,

where the computation is performed modulo $s^{3}$ since we only need the terms up to $s^{2}$. Finally, the number of integer points in the polytope $T$ is given by the sum of the coefficients of $s^{2}$ in the polynomials $\frac{N(s)}{D(s)}$, i.e, $\frac{31}{8}+\frac{17}{8}-0=6$.

\section{Our Method}

In this section we explain our method for computing enumerators of parametric polytopes based on a parametric version of Barvinok's algorithm. Since Barvinok's algorithm is vertex-based, we need to be able to compute the parametric vertices before we can apply the algorithm to parametric polytopes. We further need to compute the regions known as chambers where a fixed set of parametric vertices are "active". In Section 4.1 we first review Loechner and Wilde's algorithm [39] for computing parametric vertices and Clauss and Loechner's algorithm [14] for computing chambers. We further provide a rough complexity analysis. Section 4.2 details the actual application of Barvinok's algorithm to the parametric vertices. 


\subsection{Chamber Decomposition}

Let $P=\left\{\mathbf{x} \in \mathbb{Q}^{d} \mid A \mathbf{x} \geq B \mathbf{p}+\mathbf{c}\right\}$ be a parametric polytope of dimension $d$ with $n$ parameters. The vertices are the 0 -faces of $P$, i.e., each vertex is the unique element in the intersection $V$ of $P$ with some set $\left\{\mathbf{x} \in \mathbb{Q}^{d} \mid A^{\prime} \mathbf{x}=B^{\prime} \mathbf{p}+\mathbf{c}^{\prime}\right\}$, where $A^{\prime} \mathbf{x} \geq B^{\prime} \mathbf{p}+\mathbf{c}^{\prime}$ is a subsystem of $A \mathbf{x} \geq B \mathbf{p}+\mathbf{c}$ such that the dimension of this intersection is 0 . I.e.,

$$
V=\{\mathbf{v}\}=\left\{\mathbf{x} \in \mathbb{Q}^{d} \mid A^{\prime} \mathbf{x}=B^{\prime} \mathbf{p}+\mathbf{c}^{\prime} \wedge A^{\prime \prime} \mathbf{x} \geq B^{\prime \prime} \mathbf{p}+\mathbf{c}^{\prime \prime}\right\},
$$

where $A^{\prime \prime} \mathbf{x} \geq B^{\prime \prime} \mathbf{p}+\mathbf{c}^{\prime \prime}$ are the remaining inequalities of $P$. Since $P$ is of dimension $d$, at least $d$ equalities are needed. In fact, all vertices can be obtained by intersecting $P$ with all possible combinations of $d$ linearly independent equalities, i.e., such that $A^{\prime}$ is non-singular. The vertex $\mathbf{v}(\mathbf{p})$ is obtained as the solution of the linear system $A^{\prime} \mathbf{x}=B^{\prime} \mathbf{p}+\mathbf{c}^{\prime}$. Obviously, $\mathbf{v}(\mathbf{p})$ is a $d$-vector of affine functions of the parameters. $\mathbf{v}(\mathbf{p})$ is an active parametric vertex of $P$ only for values of the parameters where $V$ is non-empty, i.e., for the parameter values that belong to the following polyhedron:

$$
R=\left\{\mathbf{p} \in \mathbb{Q}^{n} \mid A^{\prime \prime} \mathbf{v}(\mathbf{p}) \geq B^{\prime \prime} \mathbf{p}+\mathbf{c}^{\prime \prime}\right\} .
$$

Since the number of linearly independent sets of $d$ constraints from the original set of $m$ constraints is at most $\left(\begin{array}{c}m \\ d\end{array}\right)$, the total number of parametric vertices is polynomial in the number of constraints (for fixed $d$ ) and therefore polynomial in the input size.

Example 6 Consider the following polytope:

$$
P=\left\{\left(\begin{array}{l}
i \\
j
\end{array}\right) \in \mathbb{Q}^{2} \mid i \geq 0 \wedge j \geq 0 \wedge i \leq 2 N+2 M \wedge i+2 j \leq M+2\right\} .
$$

$P$ is a parametric polytope of dimension 2. Each of its vertices is therefore given by a 0 -dimensional intersection of two of its facets. Let us compute the vertex $\mathbf{v}_{1}$ corresponding to the facets $i=2 N+2 M$ and $i+2 j=M+2$. $\mathbf{v}_{1}$ is the solution of these two equalities, i.e.,

$$
\mathbf{v}_{1}=\left(\begin{array}{c}
2 N+2 M \\
-N-\frac{M}{2}+1
\end{array}\right)
$$

The vertex $\mathbf{v}_{1}$ is active if it satisfies the remaining constraints $\{i \geq 0 \wedge j \geq 0\}$. Substituting $i=2 N+2 M$ and $j=-N-\frac{M}{2}+1$ in these constraints, we obtain the polyhedron $R_{\mathbf{v}_{1}}$, containing the parameter values for which the vertex $\mathbf{v}_{1}$ is active.

$$
R_{\mathbf{v}_{1}}=\left\{\left(\begin{array}{l}
N \\
M
\end{array}\right) \in \mathbb{Q}^{2} \mid N+M \geq 0 \wedge-2 N-M \geq-2\right\} .
$$

Proceeding similarly on the other pairs of facets, we obtain the remaining vertices:

$$
\mathbf{v}_{2}=\left(\begin{array}{c}
2 N+2 M \\
0
\end{array}\right), \mathbf{v}_{3}=\left(\begin{array}{c}
M+2 \\
0
\end{array}\right), \mathbf{v}_{4}=\left(\begin{array}{c}
0 \\
\frac{M}{2}+1
\end{array}\right) \text { and } \mathbf{v}_{5}=\left(\begin{array}{l}
0 \\
0
\end{array}\right)
$$




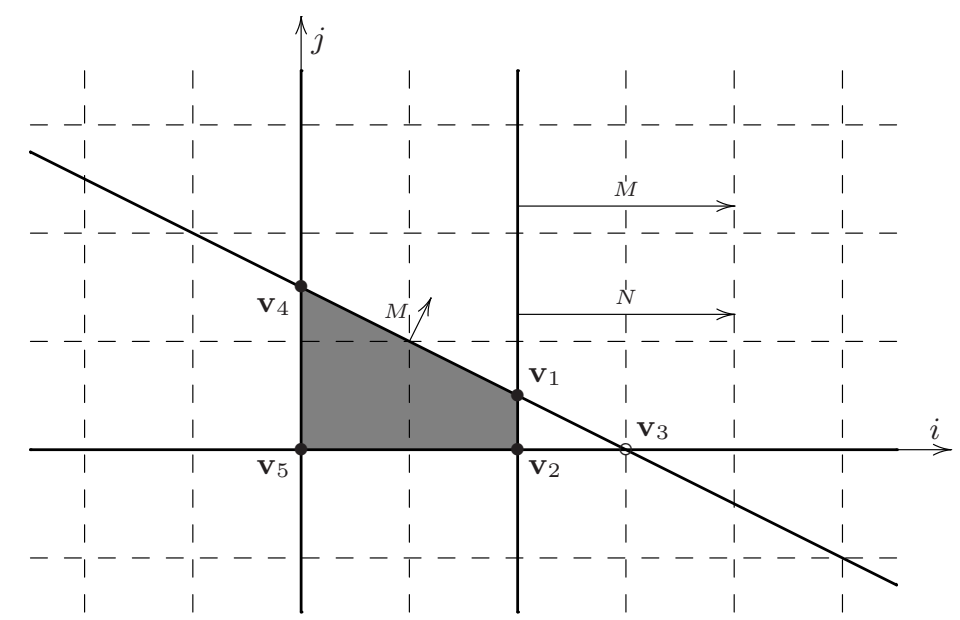

Fig. 4 The vertices of the parametric polytope from Example 6 for $N=0$ and $M=1$. The arrows show the distance over which each parametric constraint is shifted when either of the parameters is increased by one.

These vertices are active for the parameter values that belong to the polyhedra $R_{\mathbf{v}_{2}}, R_{\mathbf{v}_{3}}, R_{\mathbf{v}_{4}}$ and $R_{\mathbf{v}_{5}}$ respectively, with

$$
\begin{aligned}
& R_{\mathbf{v}_{2}}=\left\{\left(\begin{array}{l}
N \\
M
\end{array}\right) \in \mathbb{Q}^{2} \mid N+M \geq 0 \wedge-2 N-M \geq-2\right\}, \\
& R_{\mathbf{v}_{3}}=\left\{\left(\begin{array}{l}
N \\
M
\end{array}\right) \in \mathbb{Q}^{2} \mid M \geq-2 \wedge 2 N+M \geq 2\right\}, \\
& R_{\mathrm{v}_{4}}=\left\{\left(\begin{array}{l}
N \\
M
\end{array}\right) \in \mathbb{Q}^{2} \mid N+M \geq 0 \wedge M \geq-2\right\}, \\
& R_{\mathbf{v}_{5}}=\left\{\left(\begin{array}{l}
N \\
M
\end{array}\right) \in \mathbb{Q}^{2} \mid N+M \geq 0 \wedge M \geq-2\right\} \text {. }
\end{aligned}
$$

Note that $R_{\mathbf{v}_{1}}=R_{\mathbf{v}_{2}}=: R_{1}, R_{\mathbf{v}_{4}}=R_{\mathbf{v}_{5}}=: R_{3}$ and $R_{3}=R_{1} \cup R_{2}$ with $R_{2}:=R_{\mathrm{v}_{3}}$. Figure 4 shows the polyhedron $P$ and its vertices when $N=0$ and $M=1$. Note that $\mathbf{v}_{3}$ is not active here since $(0,1) \notin R_{\mathbf{v}_{3}}$. Figure 5 shows the regions in the parameter space where the respective vertices are active. The point $(0,1)$ in this figure corresponds to the configuration in Figure 4.

The regions in the parameter space $\mathbb{Q}^{n}$ with a fixed set of parametric vertices, i.e., the chambers, form the elements of a subdivision of (part of) the parameter space. Any intersection of two elements of a subdivision is also part of the subdivision. We are only interested in the full-dimensional chambers here, i.e., those of dimension $n$.

Clauss and Loechner's algorithm for computing full-dimensional chambers maintains a list $\left(R_{i}\right)$ of $n$-dimensional regions, with pairwise intersections of dimension at most $n-1$. Each of these regions $R_{i}$ has an associated list of parametric vertices $\mathcal{V}_{R_{i}}$ that are active on the region. Initially, the list contains a single pair 




Fig. 5 Regions where the vertices of the parametric polytope from Example 6 are active.

$\left(R_{\mathbf{v}_{1}},\left\{\mathbf{v}_{1}\right\}\right)$ of a particular parametric vertex and its activity region. In each step, a new vertex $\mathbf{v}_{j}$ with corresponding activity region $R_{\mathbf{v}_{j}}$ is considered. For each $R_{i}$ in the current list, if $\operatorname{dim}\left(R_{i} \cap R_{\mathbf{v}_{j}}\right)=n$ then $\left(R_{i}, \mathcal{V}_{R_{i}}\right)$ is replaced by two new pairs $\left(R_{i} \cap R_{\mathbf{v}_{j}}, \mathcal{V}_{R_{i}} \cup\left\{\mathbf{v}_{j}\right\}\right)$ and $\left(\overline{R_{i} \backslash R_{\mathbf{v}_{j}}}, \mathcal{V}_{R_{i}}\right)$, where $\bar{S}$ is the closure of $S$. After scanning the whole list, the pair $\left(\overline{R_{\mathbf{v}_{j}} \backslash \bigcup_{i} R_{i}},\left\{\mathbf{v}_{j}\right\}\right)$ is added. If any of the set differences is empty, the corresponding pair is discarded. Note that some of the intermediate regions may not be polyhedra but rather unions of polyhedra.

Example 7 Let us calculate the chambers of the polytope $P$ from the previous example. The initial list is simply

$$
\left(\left(R_{1},\left\{\mathbf{v}_{1}\right\}\right)\right) .
$$

Since $R_{\mathbf{v}_{2}}=R_{1}$ and $R_{1} \backslash R_{1}$ is obviously empty, the first iteration yields

$$
\left(\left(R_{1},\left\{\mathbf{v}_{1}, \mathbf{v}_{2}\right\}\right)\right) .
$$

As $R_{\mathbf{v}_{3}}=R_{2}$ and $\operatorname{dim}\left(R_{1} \cap R_{2}\right)<2$, the next iteration simply adds the pair $\left(R_{2},\left\{\mathbf{v}_{3}\right\}\right)$. The third iteration yields

$$
\left(\left(R_{1},\left\{\mathbf{v}_{1}, \mathbf{v}_{2}, \mathbf{v}_{4}\right\}\right),\left(R_{2},\left\{\mathbf{v}_{3}, \mathbf{v}_{4}\right\}\right)\right) .
$$

There are no additional pairs since all the set differences are empty. The final step is similar to the third and so the final result consists of two chambers $C_{1}=R_{1}$ and $C_{2}=R_{2}$, with corresponding sets of active vertices $\mathcal{V}_{C_{1}}$ and $\mathcal{V}_{C_{2}}$ :

$$
\begin{aligned}
&\left(C_{1}=\left\{\left(\begin{array}{l}
N \\
M
\end{array}\right)\right.\right.\left.\in \mathbb{Q}^{2} \mid N+M \geq 0 \wedge-2 N-M \geq-2\right\} \\
&\left.\mathcal{V}_{C_{1}}=\left\{\left(\begin{array}{c}
2 N+2 M \\
-N-\frac{M}{2}+1
\end{array}\right),\left(\begin{array}{c}
2 N+2 M \\
0
\end{array}\right),\left(\begin{array}{c}
0 \\
\frac{M}{2}+1
\end{array}\right),\left(\begin{array}{l}
0 \\
0
\end{array}\right)\right\}\right),
\end{aligned}
$$




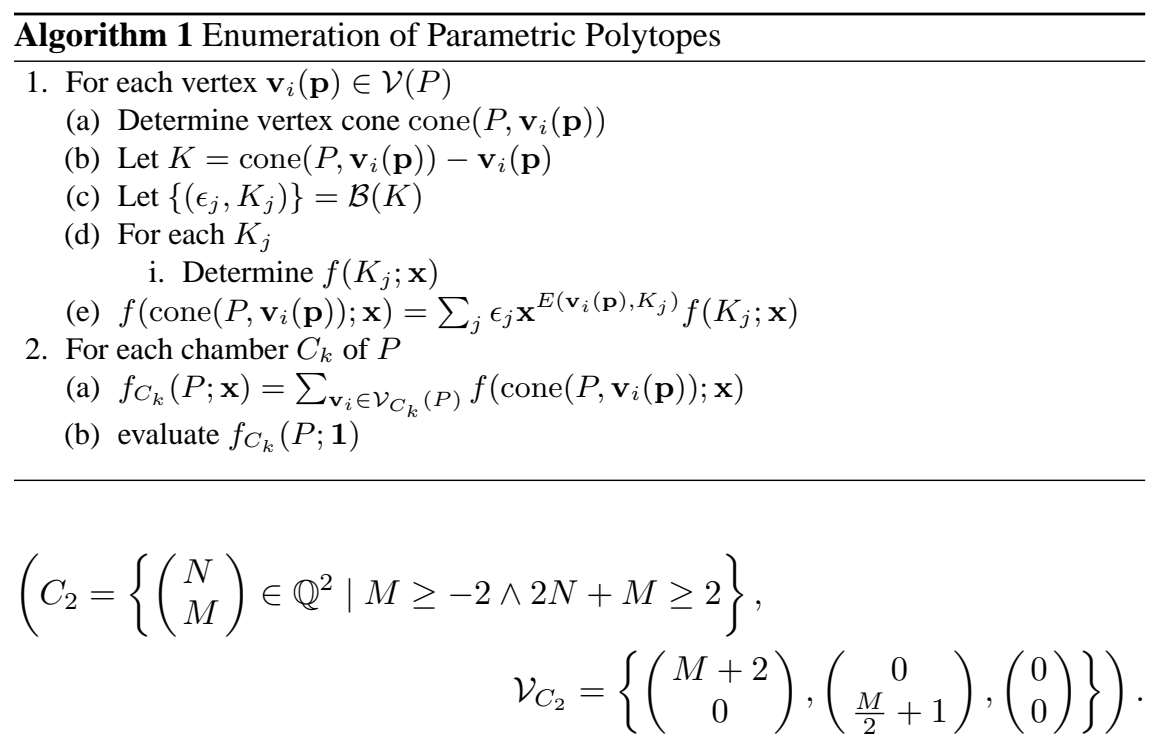

Lemma 3 The size of the chamber decomposition is polynomial in the input size (for fixed dimension) and can be computed in polynomial time.

Sketch of proof Consider the hyperplanes in the parameter space formed by the affine hulls of the $(n-1)$-dimensional intersections of pairs of chambers. These $k$ hyperplanes divide the parameter space into a number of cells that is bounded by a polynomial in $k$ (for fixed dimensions) [20]. Since (part of) these cells form a subdivision of the chambers and since the $k$ hyperplanes correspond to the projections of the generic $(n-1)$-dimensional faces of $\left\{(\mathbf{x}, \mathbf{p}) \in \mathbb{Q}^{d+n} \mid A \mathbf{x} \geq B \mathbf{p}+\mathbf{c}\right\}$ onto the parameter space, which are themselves bounded in number by a polynomial in the input size (for fixed dimensions), the number of chambers and hence also the total size is polynomial in the input size (for fixed dimensions).

The number of iterations in Clauss and Loechner's algorithm is equal to the (polynomial) number of parametric vertices. In each iteration a number of operations is performed that is proportional to the length of the list of pairs of regions and vertices. This length never decreases and its final (maximal) value is equal to the (polynomial) number of chambers. The total run time is therefore polynomial in the input size.

\subsection{Computing Step-Polynomials}

Algorithm 1 shows our application of Barvinok's method to parametric polytopes. The main idea behind this generalization is to consider Clauss and Loechner's decomposition of the parameter space and to apply Barvinok's algorithm to the fixed set of (parametric) vertices that belong to each chamber. Thus, one parametric generating function is to be computed for each of these chambers. The constant term of each generating function will be a step-polynomial. 
The generating function for the parametric polytope $P_{\mathbf{p}}$ on chamber $C$ is the parametric version of Equation (10):

$$
f_{C}\left(P_{\mathbf{p}} ; \mathbf{x}\right)=\sum_{\mathbf{v}(\mathbf{p}) \in \mathcal{V}_{C}\left(P_{\mathbf{p}}\right)} \sum_{i=1}^{\left|\mathcal{B}\left(K_{\mathbf{v}}\right)\right|} \epsilon_{i} \frac{\mathbf{x}^{E\left(\mathbf{v}(\mathbf{p}), K_{i}\right)}}{\prod_{j=1}^{d}\left(1-\mathbf{x}^{\mathbf{u}_{j}^{i}}\right)},
$$

with $\mathbf{v}(\mathbf{p})$ a parametric vertex of the polytope $P_{\mathbf{p}}$, where each coordinate of $\mathbf{v}(\mathbf{p})$ is an affine function of the parameters. $\mathcal{B}(K)=\left\{\left(\epsilon_{i}, K_{i}\right)\right\}$, with $\epsilon_{i} \in\{-1,1\}$ and $K_{i}$ a unimodular cone, is the signed unimodular decomposition of cone $K_{\mathbf{v}}$, the translation to the origin of the vertex cone at $\mathbf{v}(\mathbf{p})$. The vertex cone is again defined by the constraints that $\mathbf{v}(\mathbf{p})$ saturates, i.e., the constraints $\langle\mathbf{a}, \mathbf{x}\rangle \geq\langle\mathbf{b}, \mathbf{p}\rangle+c$ such that $\langle\mathbf{a}, \mathbf{v}(\mathbf{p})\rangle=\langle\mathbf{b}, \mathbf{p}\rangle+c$. The correctness of (15) follows from the fact that the denominators of the rational functions $f\left(K_{i} ; \mathbf{x}\right)(8)$ only depend on the elements of the vectors a of these constraints [3, page 106] and are therefore independent of the parameters.

The exponent in the numerators of (15), which corresponds to the uniquely defined point inside the translated unimodular cone, is given by the parametric version of (9):

$$
E\left(\mathbf{v}(\mathbf{p}), K_{i}\right)=\sum_{j=1}^{d}\left\lceil\lambda_{j}(\mathbf{p})\right\rceil \mathbf{u}_{j}^{i}=-\sum_{j=1}^{d}\left\lfloor-\lambda_{j}(\mathbf{p})\right\rfloor \mathbf{u}_{j}^{i},
$$

where the $\lambda_{j}(\mathbf{p})$ s are rational affine functions of the parameters that solve $\mathbf{v}(\mathbf{p})=$ $\sum_{j=1}^{d} \lambda_{j}(\mathbf{p}) \mathbf{u}_{j}^{i}$.

As in the non-parametric case, we can obtain the value of the parametric generating function (15) at $\mathrm{x}=\mathbf{1}$ by computing the appropriate coefficients in the Laurent series of each term. Again, the variable substitution proposed by Yoshida [18] is independent of the numerator and hence of the parameters. Substituting (16) in (11), we obtain

$$
N_{\mathbf{p}}(s)=(s+1)^{\left\langle\boldsymbol{\mu}, E\left(\mathbf{v}(\mathbf{p}), K_{i}\right)\right\rangle-c}=(s+1)^{\Lambda(\mathbf{p})},
$$

with $\Lambda(\mathbf{p})$ a degree-1 step-polynomial, i.e., a rational linear combination of the lower integer parts of rational affine expressions in the parameters. The coefficients of $N_{\mathbf{p}}(s)$ up to that of $s^{d}$ (i.e., those required to compute the coefficient of $s^{d}$ in $\left.N_{\mathbf{p}}(s) / D(s)\right)$ are

$$
n_{i}(\mathbf{p})=\left(\begin{array}{c}
\Lambda(\mathbf{p}) \\
i
\end{array}\right)=\frac{\prod_{j=0}^{i-1}(\Lambda(\mathbf{p})-j)}{i !} \quad \text { for } 0 \leq i \leq d .
$$

Each coefficient $n_{i}(\mathbf{p})$ in the above formula is given by a product of at most $d$ degree-1 step-polynomials. This implies that each of these coefficients is a degree$d$ step-polynomial, i.e., it is a quasi-polynomial of degree $d$ (Lemma 2). Since the coefficient of $s^{d}$ in $N_{\mathbf{p}}(s) / D(s)$ is a linear combination of these $n_{i}(\mathbf{p})$, it conforms to the same property and so does the signed sum of all these terms. That is, the value of (15) at $\mathbf{x}=1$, which is equal to the parametric number of points in $P_{\mathbf{p}}$, is a quasi-polynomial $\mathcal{E}_{C}(P ; \mathbf{p})=f_{C}\left(P_{\mathbf{p}} ; \mathbf{1}\right)$, as expected. 
Example 8 Consider the polytope

$$
P_{(p)}=\left\{\mathbf{x} \mid x_{1} \geq 0 \wedge x_{2} \geq 0 \wedge 2 x_{1}+2 x_{2} \leq p\right\}
$$

This is a parametric version of the polytope in Examples 3 and 5 where $p$ was set to 4 . This polytope has a single chamber $p \geq 0$ with parametric vertices $(0,0),\left(\frac{p}{2}, 0\right)$ and $\left(0, \frac{p}{2}\right)$. The generators of the corresponding vertex cones are $(1,0),(0,1),(-1,0),(-1,1)$ and $(0,-1),(1,-1)$ respectively. Since all these vertex cones are unimodular, there is a single term in $(15)$ for each vertex. Since $\left(\frac{p}{2}, 0\right)=-\frac{p}{2}(-1,0)+0(-1,1)$, we conclude from $(16)$ that the exponent in the numerator of the term for this vertex is

$$
\left\lceil-\frac{p}{2}\right\rceil(-1,0)=\left(\left\lfloor\frac{p}{2}\right\rfloor, 0\right) .
$$

As in Example 5, we take $\boldsymbol{\mu}=(1,-1)$, a vector not orthogonal to any of the generators. The exponent of $(s+1)$ in $(17)$ is

$$
\left\langle(1,-1),\left(\left\lfloor\frac{p}{2}\right\rfloor, 0\right)\right\rangle+3=\left\lfloor\frac{p}{2}\right\rfloor+3,
$$

where $c=-3$ as in (13). The other vertices are handled similarly and we obtain

$$
f(P ; s)=\frac{(s+1)^{\left\lfloor\frac{p}{2}\right\rfloor+3}}{s^{2}(s+2)}+\frac{(s+1)^{-\left\lfloor\frac{p}{2}\right\rfloor}}{s^{2}(s+2)}-\frac{s+1}{s^{2}}
$$

where we simplified the denominators that we already calculated in (13). To evaluate $f(P ; 0)$, we calculate the coefficient of $s^{2}$ in

$$
\frac{N(s)}{D(s)}=\frac{(s+1)^{\left\lfloor\frac{p}{2}\right\rfloor+3}+(s+1)^{-\left\lfloor\frac{p}{2}\right\rfloor}}{s+2}-(s+1) .
$$

The second term does not contribute to $s^{2}$. The terms in the numerator of the first term can be expanded according to (18) as

$$
(s+1)^{\left\lfloor\frac{p}{2}\right\rfloor+3} \equiv 1+\left(\left\lfloor\frac{p}{2}\right\rfloor+3\right) s+\left(\frac{1}{2}\left\lfloor\frac{p}{2}\right\rfloor^{2}+\frac{5}{2}\left\lfloor\frac{p}{2}\right\rfloor+3\right) s^{2} \bmod s^{3}
$$

and

$$
(s+1)^{-\left\lfloor\frac{p}{2}\right\rfloor} \equiv 1-\left\lfloor\frac{p}{2}\right\rfloor s+\left(\frac{1}{2}\left\lfloor\frac{p}{2}\right\rfloor^{2}+\frac{1}{2}\left\lfloor\frac{p}{2}\right\rfloor\right) s^{2} \bmod s^{3} .
$$

while the denominator is given in (14). The coefficient of $s^{2}$ in (19) is therefore

$$
\mathcal{E}(P ; p)=f(P ; 0)=\frac{1}{2}\left\lfloor\frac{p}{2}\right\rfloor^{2}+\frac{3}{2}\left\lfloor\frac{p}{2}\right\rfloor+1 \text {. }
$$


Note that in contrast to Clauss's method (see Section 6.2.1) our algorithm computes a quasi-polynomial for any chamber, no matter its size or shape. Furthermore, each quasi-polynomial is calculated analytically, which enables us to avoid the use of exponentially-sized lookup-tables to represent periodic numbers (see Section 6.2.2). Since Barvinok's decomposition is polynomial in the input size [4] and since the number of parametric vertices is also polynomial (for fixed dimensions) [39], the number of distinct affine expressions that appear in the final step-polynomial for any given chamber, i.e., those that originate from expressions (16), is polynomial in the input size. Since the degree of this final polynomial is fixed, its total size is polynomial in the input size (for fixed dimension). According to Lemma 3, the size of the chamber decomposition is also polynomial and we have the following proposition.

Proposition 2 The size of the enumerator of a parametric polytope is polynomial in the size of the parametric polytope (for fixed dimension) and it can be computed in polynomial time.

\section{Extensions}

Our method proves to be useful to help solving two further counting problems. The first is counting the number of points in a parametric system of inequalities with existential variables, the second is counting the number of solutions to parametric Presburger formulas. Both of these counting problems can be preprocessed (albeit in exponential time, even for fixed dimensions), to generate counting problems of the form (1), which the presented algorithm solves in polynomial time for fixed dimensions.

\subsection{Existential Variables}

The general form of the counting problems with existential variables is

$$
\#\left\{\mathbf{x} \in \mathbb{Z}^{d} \mid \exists \mathbf{y} \in \mathbb{Z}^{d^{\prime}}: A \mathbf{x}+D \mathbf{y} \geq B \mathbf{p}+\mathbf{c}\right\}
$$

Such a counting problem can be handled by preprocessing the input with PIP [22] to obtain a union of disjoint rational polyhedra that is such that the total number of integer points in these sets is equal to the number of elements in the original set $[10,49]$. This preprocessing therefore reduces the counting problem to a set of subproblems of the form (1) and the resulting enumerators can simply be summed to obtain the enumerator of the original set. Counting problems of this type occur in the program analyses described in $[1,2,10,15,29,30]$.

Example 9 Consider array reference $A[i+j]$ in Figure 1. The different array elements that are accessed by the complete execution of the loop is given by the following set:

$$
\begin{aligned}
\left\{x \in \mathbb{Z} \mid \exists(i, j) \in \mathbb{Z}^{2}:\right. & =i+j \wedge 0 \leq j \leq N-2 i \wedge \\
0 & \leq i \wedge N-M \leq i \leq N-M+3\}
\end{aligned}
$$


Parametrically counting the number of elements in this set gives the number of different array elements accessed by the loop, and our implementation gives the following answer:

$$
\begin{cases}2 N-M+1 & \text { if } M \leq N \leq 2 M \\ N+1 & \text { if } 0 \leq N \wedge M-3 \leq N \leq M-1\end{cases}
$$

\subsection{Presburger formulas}

Presburger formulas consist of linear inequalities of integer variables, combined by existential and universal quantifiers, disjunction, conjunction and negation $(\exists$, $\forall, \vee, \wedge, \neg)$. The number of solutions to parametric Presburger formulas can be counted by using the Omega calculator [32] to transform them into a disjoint union of sets of the form that appears in (20). Note that like the preprocessing step using PIP, this preprocessing step is worst-case exponential, even for fixed dimensions. Counting the number of integer solutions to parametric Presburger formulas is required in the analyses described in $[7,13,33]$.

Example 10 The forward reuse distance of a memory access to a memory location $l$ is the number of different memory locations referenced until the next access to $l$. The different memory locations between a use and the next reuse of $A[i+j]$ in Figure 1 can be expressed by the following Presburger formula [7]:

$$
\begin{aligned}
\left\{x \in \mathbb{Z} \mid \exists\left(i^{\prime \prime}, j^{\prime \prime}\right):\right. & x=i^{\prime \prime}+j^{\prime \prime} \wedge \\
& \operatorname{IS}(i, j) \wedge \operatorname{IS}\left(i^{\prime \prime}, j^{\prime \prime}\right) \wedge \\
& (i, j)<_{\operatorname{lex}}\left(i^{\prime \prime}, j^{\prime \prime}\right) \wedge \\
\neg\left(\exists\left(i^{\prime}, j^{\prime}\right):\right. & \operatorname{IS}\left(i^{\prime}, j^{\prime}\right) \wedge(i, j)<_{\text {lex }}\left(i^{\prime}, j^{\prime}\right)<_{\operatorname{lex}}\left(i^{\prime \prime}, j^{\prime \prime}\right) \\
\wedge & \left.\left.i^{\prime}+j^{\prime}=i+j\right)\right\},
\end{aligned}
$$

where

$$
\begin{aligned}
\operatorname{IS}(a, b) & \equiv 0 \leq a \wedge N-M \leq a \leq N-M+3 \wedge 0 \leq b \leq N-2 a \\
(a, b)<_{\operatorname{lex}}(c, d) & \equiv a<c \vee(a=c \wedge b<d)
\end{aligned}
$$

By counting the number of integer values of $x$, and considering $i, j, M$ and $N$ as parameters, the forward reuse distance is computed for each execution of $A[i+j]$ at iteration $(i, j)$. Our implementation gives the following answer:

$$
\{N-1-2 i \quad \text { if } N \leq i+M \leq 2+N \wedge 2 i+j<N \wedge 0 \leq i \wedge 1 \leq j .
$$

This counting problem is inherently parametric, since it requires an answer in function of the loop variables $i$ and $j$. In other words, even if $N$ and $M$ were fixed, a parametric counting problem would result. 


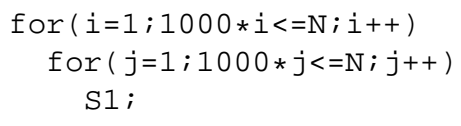

(a) How many times does $\mathrm{S} 1$ execute?

$$
\begin{cases}\frac{N^{2}}{1000000} & \text { if } 1000 \text { divides } N \\ \frac{(N-1) N}{1000000}-\frac{N-1}{1000000} & \text { if } 1000 \text { divides } N-1 \\ \cdots & \\ \frac{(N-999) N}{1000000}-\frac{999 N-998001}{1000000} & \text { if } 1000 \text { divides } N-999\end{cases}
$$

(b) Solution computed by hand using Pugh's method [42]

$$
\left\lfloor\frac{N}{1000}\right\rfloor^{2}
$$

(c) Solution generated by our method

Fig. 6 Example of an answer generated by Pugh's method. The number of different cases in Pugh's answer is as large as the factor of $i$ and $j$ in the program in (a) (1000 in this example). Therefore, the solution size of Pugh's method is exponentially large.

\section{Related Work}

Two methods are often cited for counting the number of points in a parametric polytope: Pugh (1994) [42] and Clauss and Loechner (1998) [14].

\subsection{Pugh's method}

The technique of Pugh consists of a set of simplification and rewrite rules and the application of a set of standard summation formulas for some base cases. In contrast to our technique and that of Claus and Loechner, no implementation of his technique appears to be publicly available. Furthermore, the description of the method in [42] fails to indicate which rewrite rules to use when several are applicable. We are therefore unable to systematically compare our results to those that would or would not be obtained using that method. Application by hand on the example in Figure 6 shows that any implementation would produce exponentially large solutions in the worst case, even for fixed dimensions.

\subsection{Clauss's method}

As already explained in the previous section, our technique and Clauss's method share the decomposition into chambers, but whereas the latter can produce exponentially sized quasi-polynomials or sometimes no quasi-polynomial at all, our technique always produces polynomially-sized quasi-polynomials in polynomial time (for fixed dimensions). 


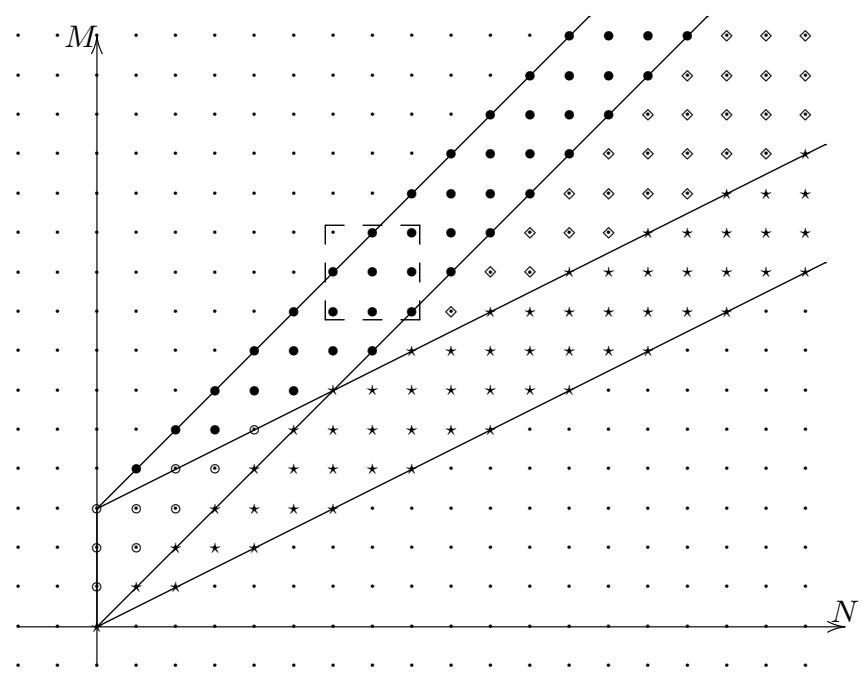

Fig. 7 Geometrical representation of the chambers of Equation (2). The points in the different chambers are shown by different symbols. The domain marked by $\bullet$ is degenerate.

6.2.1 Interpolation and Degenerate Domains Based on the knowledge of the structure of the solution (Proposition 1), Clauss and Loechner [14] calculate the number of points in a set of instances of $P_{\mathbf{p}}$ for fixed values of $\mathbf{p}$ in a given chamber, called initial countings, and then calculate the quasi-polynomial for this chamber through interpolation. During this calculation, they directly determine the elements in the lookup-tables representing the periodic numbers. To interpolate a $d$-dimensional quasi-polynomial with periods $q_{i}$ their algorithm requires $\prod_{i=1}^{n}(d+1) q_{i}$ initial countings. Since the implementation is based on repeated Vandermonde interpolation, it searches for fixed parameter values located in a hyperrectangle. However, it is not always possible to find a hyperrectangle of the correct size that is completely inside a given chamber.

For example, consider the chamber $N \leq M \leq N+3 \wedge N \leq 2 M-7$ from the polytope in Equation (2). This chamber is geometrically represented with $\bullet$ in Figure 7. For this chamber, the period in both dimensions is 1 , and the implementation of Clauss's method in PolyLib [37] searches for a solution of the following form

$$
a N^{2} M^{2}+b N^{2} M+c N^{2}+d N M^{2}+e N M+f N+g M^{2}+h M+i .
$$

To find the nine unknown values, Clauss's method looks for a $3 \times 3$ rectangle in the chamber for which it can compute initial countings for. As is clear from Figure 7, however, no such rectangle can be found and the method fails to compute the solution. The chambers where this problem occurs are known as degenerate domains. This problem could in principle be solved by considering other interpolation techniques or by adding an extra parameter [40] $]_{4}^{4}$

\footnotetext{
4 Version 5.22.0 of PolyLib, released after the initial submission of this paper, implements the technique of [40].
} 


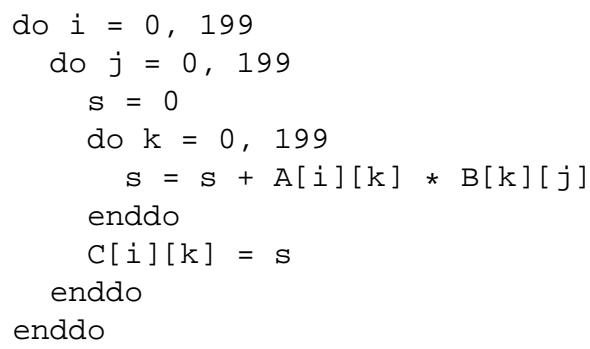

(a) Source code

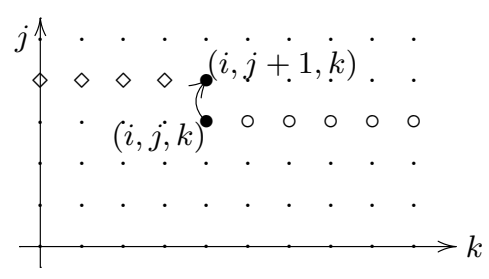

(b) Intermediate accesses

Fig. 8 Matrix multiplication

6.2.2 Large Solution Size Since the periods $q_{i}$ are bounded only by the value of the coefficients in the input, they can be exponential in terms of the input size and so the worst-case computation time for the quasi-polynomial of a single chamber is exponential even for fixed dimension. Since Clauss's method is based on the lookup-table representation of periodic numbers, the output size is also exponential in the input size.

Consider, for example, the program in Figure 8 (matrix multiplication). Suppose we want to count the number of distinct Translation Lookaside Buffer (TLB) pages accessed between two consecutive accesses to the same TLB page. This count is an indication of the number of TLB page misses that can be expected and is called the reuse distance [7].

For simplicity, we will assume that $A[i][k]$ and $B[k][j]$ access different TLB pages and we will concentrate on A [i] [ $\mathrm{k}]$. We assume that $A$ is a $200 \times$ 200 matrix, which is laid out in column major order, and starts at address zero. Furthermore, an element size of 4 bytes is assumed. As such, A [i] [ $k$ ] is located at address $4 \times(200 k+i)$.

Iterations $(i, j, k)$ and $(i, j+1, k)$ access the same array element $\mathrm{A}[\mathrm{i}][\mathrm{k}]$. Figure $8 \mathrm{~b}$ shows the iterations that are executed between these two iterations: iterations $(i, j, k+1 \ldots 199)$ ( $\circ$ on the figure) and iterations $(i, j+1,0 \ldots k-1)(\diamond$ on the figure). The set of TLB pages accessed by the o-iterations can be described as

$$
S_{1}=\left\{t \mid \exists k^{\prime}: t=\left\lfloor\frac{800 k^{\prime}+4 i}{L}\right\rfloor \wedge 0 \leq i, j, k \leq 199 \wedge k+1 \leq k^{\prime} \leq 199\right\},
$$

where $i, j$ and $k$ are parameters. Assuming page size $L=4096$, this can be written as a set of linear constraints:

$$
\begin{aligned}
S_{1}=\left\{t \mid \exists k^{\prime}: 1024 t \leq 200 k^{\prime}+\right. & i \leq 1024 t+1023 \\
& \left.\wedge 0 \leq i, j, k \leq 199 \wedge k+1 \leq k^{\prime} \leq 199\right\}
\end{aligned}
$$

and further simplified to (e.g., using Omega [32])

$$
\begin{aligned}
S_{1}=\{t \mid 0 \leq i \wedge 1024 t-39800 & \leq i \leq 199 \wedge 0 \leq k \leq 198 \\
& \wedge 0 \leq j \leq 199 \wedge i+200 k \leq 823+1024 t\} .
\end{aligned}
$$


We obtain a similar expression $S_{2}$ for the $\diamond$-iterations. The total count of TLB pages is \# $\left(S_{1} \cup S_{2}\right)=\# S_{1}+\#\left(S_{2} \backslash S_{1}\right)$. Concentrating on $S 1$, we see that it is a one-dimensional polytope and using PolyLib we can find out that its vertices are

$$
\frac{i}{1024}+25 \frac{k}{128}-\frac{823}{1024} \text { and } \frac{i}{1024}+\frac{4975}{128} \text {. }
$$

Since the dimension of this polytope is $d=1$ and the periods are $q_{i}=1024, q_{j}=$ 1 and $q_{k}=128$, the interpolation method requires $2^{3} \cdot 1024 \cdot 128$ initial countings. If we assume we need two bytes to represent a value, then we need up to $256 \mathrm{KiB}$ just to store a single periodic number in the output. Using our own technique, we obtain the following equivalent, but much shorter solution in polynomial time (for fixed dimensions):

$$
\left\lfloor\frac{i+888}{1024}\right\rfloor-\left\lfloor\frac{i+200 k+199}{1024}\right\rfloor+39 .
$$

Although the degeneracy problem can in principle be solved, the problem of a worst-case exponentially-sized output is intrinsic to this approach.

A number of the proposed compiler methods $[7,25,38,48]$ hard-code the resulting quasi-polynomial in the program they are optimizing. For these optimizations, large quasi-polynomials result in large binaries, making the optimizations less interesting, especially in an embedded systems context. Using our method, the size of the resulting quasi-polynomials does not grow exponentially.

\subsection{Other Techniques}

Although LattE initially only counted the number of points in non-parametric polytopes, it has been extended to handle some form of parametric polytopes by what the authors call the homogenized Barvinok algorithm [16]. They only support a single parameter $p$, however. In particular, they only consider dilations $p P$ of a non-parametric polytope $P$ by a factor $p$. This means that all the vertices (see Eq. (5)) are of the form $p \mathbf{v}$ and that they do not need to consider chambers, since the limited set of problems they handle always have a single fixed chamber. The quasi-polynomials that correspond to these enumeration problems are called Ehrhart quasi-polynomials. Rather than computing the Ehrhart quasi-polynomials, however, they compute Ehrhart series, which are formal power series closely related to Ehrhart quasi-polynomials and which seem to be more appropriate for their application domain. The main difference is that in an Ehrhart series $\sum c_{p} t^{p}$, the number of points $c_{p}$ in the dilatation $p P$ is equal to the coefficient of the term $t^{p}$.

Beck [5] describes a general technique for computing vector partition functions. In principle, the enumeration of any parametric polytope can be computed as the vector partition function of a higher dimensional problem. He does not provide a complexity analysis, though, but his technique is based on partial fractions and standard techniques for computing partial fractions [31] are exponential, even for fixed dimensions. 
Recently some advances have been made towards automata-based counting [9,41]. Although these techniques handle a larger class of problems (solutions to Presburger formulas), they do not support symbolic parameters. Preliminary experiments have shown that in the intersection of the application domains, i.e., for non-parametric polytopes, our method is as fast or faster (up to a factor 100 in some exceptional cases) than Parker's, except for polytopes with a large number (say thousands) of vertices. For such polytopes, the homogenized Barvinok algorithm as implemented by Latt $\mathrm{E}$ would be more appropriate. 5

\section{Implementation Details}

In Section 4, we assumed that $P$ is full-dimensional. If $P$ is not full-dimensional, then we transform it into another polytope which has the same number of points but which is embedded in a lower dimensional space. In particular, the ambient space of the transformed polytope has the same dimension as $P$ and so the transformed polytope is full-dimensional. If $P$ is of dimension $d-l$, with $l \geq 1$, then its description contains an equality $\langle\mathbf{a}, \mathbf{x}\rangle=\langle\mathbf{b}, \mathbf{p}\rangle+c$. Let $\mathbf{a}^{\prime}=\mathbf{a} / g$, with $g$ the greatest common divisor (gcd) of the elements in $\mathbf{a} . \mathbf{a}^{\prime T}$ can be extended to a unimodular matrix $U[8] .6$ Let $P^{\prime}=U P$. Since $U$ and $U^{-1}$ are unimodular, there is a one-to-one correspondence between the integer points in $P$ and those in $P^{\prime}$ and so the number of points in both polytopes is the same, i.e., $\mathcal{E}\left(P^{\prime}\right)=\mathcal{E}(P)$. Furthermore, the first coordinate $x_{1}^{\prime}$ of $P^{\prime}$ is independent of the other coordinates since $g x_{1}^{\prime}=g\left\langle\mathbf{a}^{\prime}, \mathbf{x}\right\rangle=\langle\mathbf{b}, \mathbf{p}\rangle+c$ by construction of $U$ and so $P^{\prime}$ is the product of $P^{\prime \prime}=\{(\langle\mathbf{b}, \mathbf{p}\rangle+c) / g\}$ and some $P_{1} \in \mathbb{Q}^{d-1}$. Therefore we can factor $P$ and compute the number of points in $P$ as the product of those in $P^{\prime \prime}$ and $P_{1}$, i.e., $\mathcal{E}(P)=\mathcal{E}\left(P^{\prime}\right)=\mathcal{E}\left(P^{\prime \prime}\right) \cdot \mathcal{E}\left(P_{1}\right)$. The number of integer points in $P^{\prime \prime}$ is zero or one depending on the parameters and this number can be represented by a periodic number. Repeating the above $l$ times yields a $P_{l} \in \mathbb{Q}^{d-l}$ of full dimension.

Even if $P$ is full-dimensional, then we may in some cases still be able to write it as a product of two or more sets [28]. Since the dimension of each set is smaller than that of $P$, we can greatly reduce the computation time by calculating the number of points in each factor separately and multiplying the results afterward. Note that we also need to "multiply" the chambers, i.e., the chambers of the product are the common refinement of the corresponding chambers in the factors. Furthermore, if $P$ or one of its factors is one-dimensional then it has two vertices $l(\mathbf{p}) \leq u(\mathbf{p})$ and we simply calculate $\lfloor u(\mathbf{p})\rfloor-\lceil l(\mathbf{p})\rceil+1$ (again a periodic number) in each chamber rather than using the algorithm of Section 4 .

We can also apply factorization if $P$ itself does not factor but instead some unimodular transformation of $P$. Again, such a unimodular transformation does not affect the number of integer points. To see which unimodular transformation we should apply, we first need to explain how we can detect the factors of a polytope. If we can group the elements of $\mathbf{x}$ into two subsets $\mathrm{x}^{\prime}$ and $\mathrm{x}^{\prime \prime}$ such that none of

\footnotetext{
5 This version embeds a polytope $P$ in a single cone with a polar that has few if $P$ has few facets [18].

${ }^{6}$ A unimodular matrix is an integer matrix with determinant 1 or -1 .
} 
the inequalities defining $P$ have non-zero coefficients for elements of both subsets, then we can factor $P$ along these two subsets. We can maximize the number of disjoint such groups by computing the Hermite normal form of a full row rank $d \times d$ subsystem $A^{\prime}$ of $A$, with $P=\left\{\mathbf{x} \in \mathbb{Q}^{d} \mid A \mathbf{x} \geq \mathbf{c}\right\}$. The Hermite normal form $H$ of a full row rank (square) matrix $A^{\prime}$ is the unique lower triangular, nonnegative matrix with each element of the diagonal strictly larger than the other elements of the same row and such that $H=A^{\prime} U$ for some unimodular matrix $U$. Note that we can indeed find a full row rank subsystem since otherwise $P$ would contain a line. The Hermite normal form maintains the decomposability into subgroups since we can also compute this form for each of the blocks in the block matrix $A^{\prime}$ separately and obtain the same (unique) normal form for the whole matrix. Applying the same unimodular transformation to the remaining rows of $A$, we see that the groups of columns remain disjoint. No other unimodular transformation can therefore result in more disjoint groups.

Our procedure for calculating Barvinok's unimodular cone decomposition is an independent reimplementation of the corresponding procedure in Latt $\mathrm{E}$ [17] as described in [18]. Like LattE, we use Shoup's implementation [44] of Lenstra, Lenstra and Lovasz' basis reduction algorithm and GMP [26] for computing in exact long integer arithmetic. Unlike LattE, however, we use PolyLib [37] for performing polyhedral operations, since this allows us to reuse the procedures for subdividing the parameter space into chambers [39]. The disadvantage of using PolyLib is that it incurs a speed penalty by insisting on maintaining the dual representation for all polytopes and by its non-optimal use of the GMP library.7

\section{Experiments}

Our experiments were performed on an otherwise idle 2.66GHz Pentium 4 machine running Linux using version 0.13 of our own barvinok library and version 5.21 .0 of PolyLib, ${ }^{8}$ The latter implements Clauss's method and, as explained in the previous section, is also used by our implementation for performing polyhedral operations. Furthermore, our implementation uses the same data structures to store polynomials. The only difference is in the representation of periodic numbers. Quasi-polynomials are stored by first rewriting each lower integer part as the difference of the affine expression and its fractional part. Polynomials of these fractional parts then form the periodic numbers. The size measure corresponds to the total memory size allocated to store the result. Note that the granularity is 4 bytes, since the experiments were performed on a 32 bits machine.

During the past years, a number of researchers have observed degeneracy problems in Clauss's method while they were trying to count the number of points in the polytopes generated by their compiler analyses. Thanks to their collaboration,

\footnotetext{
7 Version 5.22.0 of PolyLib can avoid the computation of the dual in some cases.

8 Version 0.13 did not implement the full factorization of Section 7, but rather only factors out (straight) prisms. Full factorization has little effect on the polytopes that appear in the experiments of this section.
} 


\begin{tabular}{|l|r|r|r|r|}
\hline program & $\begin{array}{r}\text { nr. } \\
\text { of } \\
\text { poly- }\end{array}$ & \multicolumn{2}{|c|}{$\begin{array}{c}\text { Clauss's } \\
\text { method }\end{array}$} & $\begin{array}{r}\text { our } \\
\text { method }\end{array}$ \\
\cline { 3 - 5 } & $\begin{array}{r}\text { \#degen. } \\
\text { topes }\end{array}$ & $\begin{array}{r}\text { exec. } \\
\text { domains }\end{array}$ & $\begin{array}{r}\text { exec. } \\
\text { time }\end{array}$ \\
\hline time
\end{tabular}

Table 1 Number of polytopes constructed by reuse distance calculation, number of degenerate domains using Clauss's method, and execution time of Clauss's and our method. The numbers marked by an * are partial since they only apply to the non-degenerate domains.

we have collected polytopes resulting from cache miss equations [27], exact analysis of cache behavior [13], converting software to hardware implementations [48], computing reuse distance equations [7] as well as some as yet unpublished compiler analyses. For all these polytopes on which the implementation of Clauss's method in PolyLib version 5.21.0 fails, our method successfully computes the solution. Our implementation and a set of such polytopes are available from http://freshmeat. net/projects/barvinok/.

Furthermore, we made a more extensive evaluation by comparing the results, the computation time and the size of the solution for parametric polytopes resulting from the following three analyses:

- Computation of reuse distances [7]. The reuse distance of a memory access to an array element $a$ is the number of different array elements that are fetched since the previous access to $a$. First, the array elements accessed between use and reuse are represented by a Presburger formula and converted to a union of parametric polytopes using Omega [32]. Then, the reuse distance is computed by symbolically counting the number of integer points in the polytopes. In our experiments, none of these polytopes have periodic behavior and therefore their period is always 1 . The solution size is then polynomial for both fractional parts and table representation of periodic numbers. However, Table 1 shows that for 4 out of the 7 programs for which reuse distances are calculated, Clauss's method fails to compute the reuse distance, due to degenerate domains. Thanks to our method, the reuse distance can be calculated for all programs, which makes calculation of reuse distances practical and potentially useful to implement in industrial compilers. Furthermore, Table 1 shows that the computation times are reasonable and compared to Clauss's method, our method is about twice as fast for this set of polytopes. For $99 \%$ of these polytopes, there is only a single chamber. The remaining $1 \%$ have two to four chambers. The dimension $d$ of these polytopes is 1 or 2 , whereas the number of parameters ranges between 2 and 7 . 

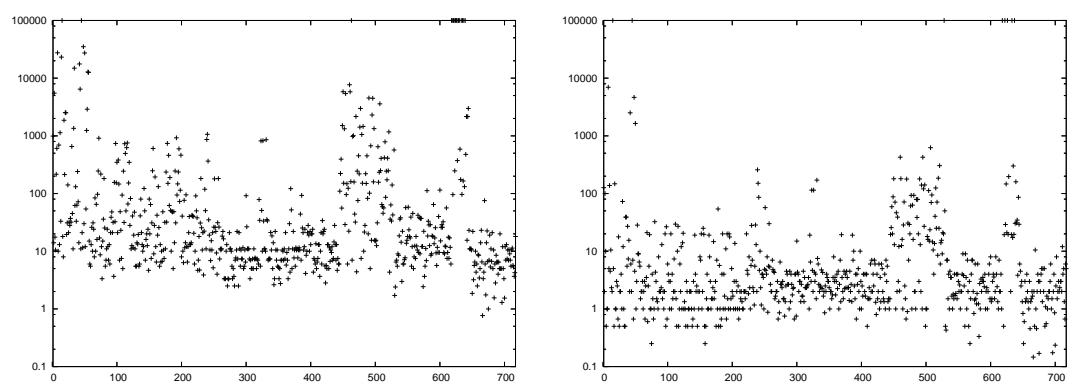

Fig. 9 Execution time ratio for Clauss's method compared to ours for the original polytopes on the left and preprocessed polytopes on the right.

- Computation of reuse distances over cache lines. The basic idea is the same as the one behind the previous experiment, except that the reuse distance is measured between successive accesses to the same cache line instead of the same array element. The resulting Presburger formulas are more complicated in this case and after simplification by Omega, the resulting sets may still contain existentially quantified variables. These variables can be removed using a technique proposed by Boulet and Redon [10] based on parametric integer programming [22]. A direct application of this technique results in parametric polytopes with at least as many equalities as there are existential variables. Since the implementation of Clauss's method does not appear to exploit these equalities, we report on experiments on both the original and preprocessed versions with the equalities removed. From a total of 18951 polytopes, 907 had a computation time of more than $0.1 \mathrm{~s}$. The implementation of Clauss's method failed to produce a complete result for 190 of these polytopes, due to "degenerate domains". The ratio of the execution times for the remaining polytopes is shown for the "raw" polytopes on the left of Figure 9 and for the polytopes with equalities removed on the right. The horizontal axis identifies the individual polytopes. For 17 polytopes on the left and 8 polytopes on the right, the computation with Clauss's method exceeds 10 minutes. The "ratio" for these polytopes is fixed to 100000 on the figures. For $76 \%$ of these polytopes, there is only a single chamber. The remaining $24 \%$ have two to eight chambers. The total dimension $d+n$ of these polytopes ranges between 0 and 7 (after removal of equalities), whereas the number of parameters $n$ ranges between 1 and 3. For more information on this experiment, we refer to [49].

- Computation of the number of cache lines and the number of memory pages accessed by a reference in a given execution of a loop nest [23]. In contrast to the polytopes resulting from the first experiment, some of these polytopes do have large periods. In Figure 10, the calculation time is plotted in function of the periodic behavior. In Figure 11, the size of the solution is shown as a function of the period of the solution. Remember that the period itself is exponential in the input size, even for fixed dimensions. Figure 10 shows that the computation time increases sharply with the period for Clauss's method. On the other hand, the computation time of our method is always less than 1 


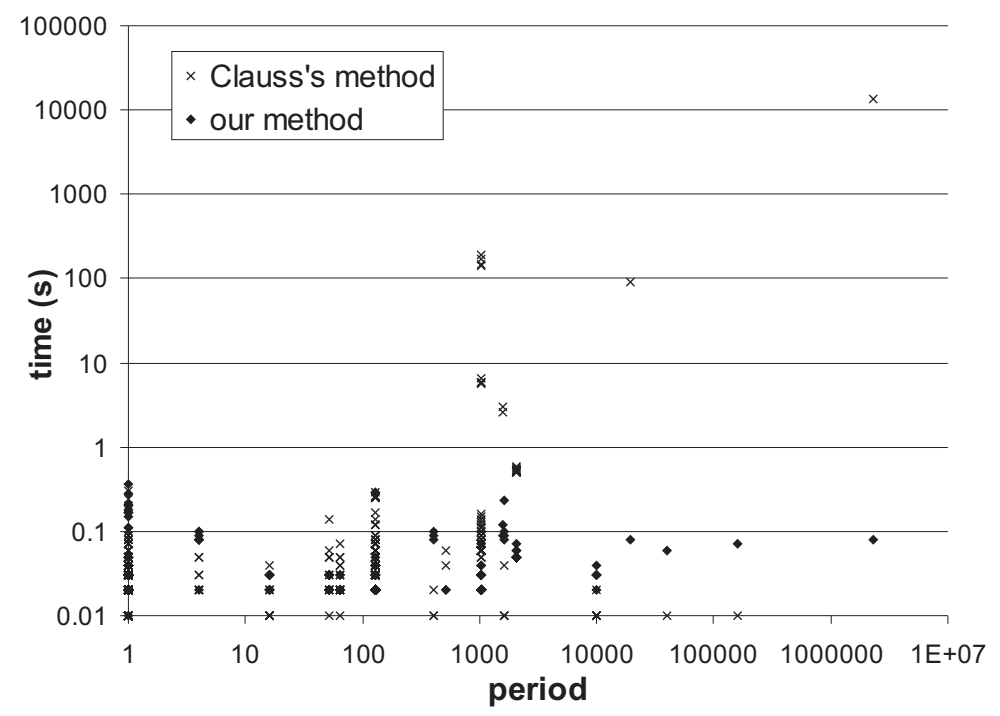

Fig. 10 Execution time as a function of maximum period of computed polynomials.

second, irrespective of the period. In comparison, the computation time using Clauss's method increases to more than 3 hours for one polytope. Figure 11 shows that the size of the solution using Clauss's method can increase to more than $32 \mathrm{MiB}$, whereas our method always produces solution sizes smaller than $9 \mathrm{KiB}$. For $99 \%$ of these polytopes, there is only a single chamber. The remaining $1 \%$ have two chambers. The dimension $d$ of these polytopes is 1 or 2 , whereas the number of parameters ranges between 0 and 3 .

\section{Conclusion and Future Work}

Many compiler analyses and optimizations require the computation of the number of integer solutions to parametric systems of linear inequalities. This count can be represented by a set of quasi-polynomials, each valid in part of the parameter space. We have presented a new method, based on Barvinok's decomposition, for constructing these quasi-polynomials analytically, resulting in the first implementation that handles all parametric polytopes of the form (1). By further using an alternative representation using lower integer parts, we compute polynomially sized quasi-polynomials - crucial for compiler optimizations that encode the quasi-polynomial in the resulting optimized program-in polynomial time, making a whole class of existing optimization techniques practically usable.

Our quasi-polynomials, though polynomially sized (for fixed dimensions), can in some cases still be relatively large. Further research into simplification of steppolynomials beyond what is applied already may reduce their size even further. Although Presburger formulas can in principle be handled by first converting to 


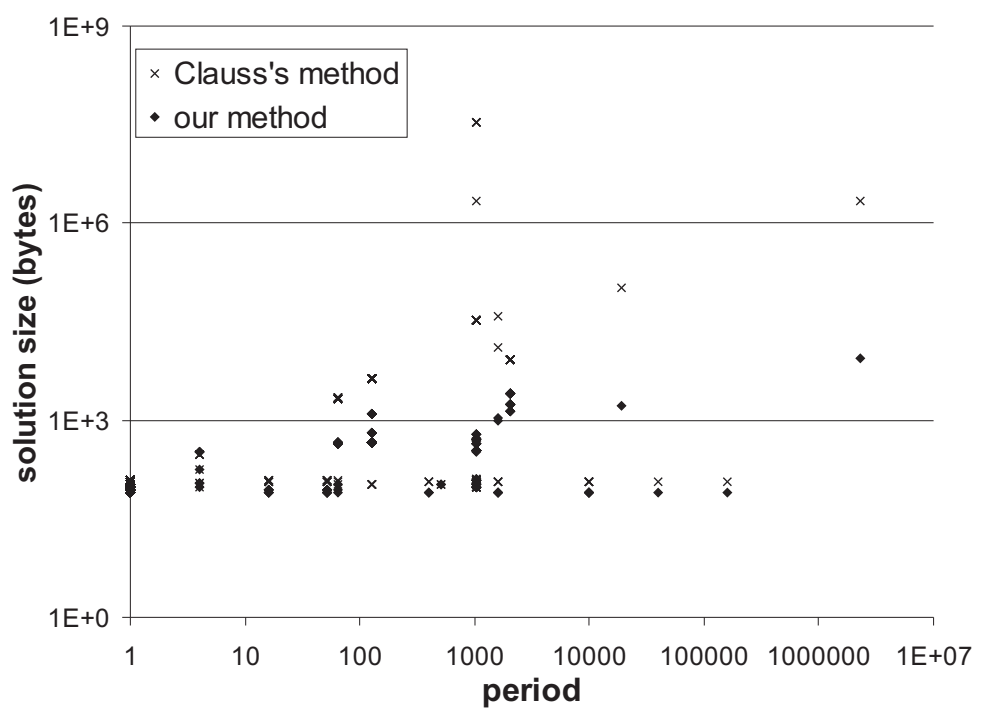

Fig. 11 Solution size as a function of maximum period of computed polynomials.

disjoint disjunctive normal form using Omega and then removing the existential variables that may remain using PIP, both of these preprocessing techniques are worst-case exponential, even for fixed dimension. We therefore plan to extend our technique to counting the number of points in integer projections of parametric polytopes in polynomial time.

Acknowledgements We thank Jesús A. De Loera, Jörg Rambau and the anonymous reviewers of both this paper and [50] for their advice and Erin Parker for providing us with an implementation of her technique.

Kristof Beyls was supported by research projects GOA-12051002 and IWT-SB991147. Sven Verdoolaege was supported by FWO-Vlaanderen.

\section{References}

1. S. Anantharaman and S. Pande. Compiler optimizations for real time execution of loops on limited memory embedded systems. In The 19th IEEE Systems Symposium (RTSS98), 1998.

2. F. Balasa, F. Catthoor, and H. De Man. Background memory area estimation for multidimensional signal processing systems. IEEE Transactions on Very Large Scale Integration (VLSI) Systems, 3(2):157-172, June 1995.

3. A. Barvinok and J. Pommersheim. An algorithmic theory of lattice points in polyhedra. New Perspectives in Algebraic Combinatorics, 38:91-147, 1999.

4. A. I. Barvinok. A polynomial time algorithm for counting integral points in polyhedra when the dimension is fixed. In 34th Annual Symposium on Foundations of Computer Science, pages 566-572. IEEE, Nov. 1993. 
5. M. Beck. The partial-fractions method for counting solutions to integral linear systems. Discrete Comp. Geom., 32:437-446, 2004. (special issue in honor of Louis Billera).

6. M. Bednara, F. Hannig, and J. Teich. Generation of distributed loop control. In In Embedded Processor Design Challenges: Systems, Architectures, Modeling, and Simulation (SAMOS), volume 2268 of Lecture Notes in Computer Science, pages 154-170, 2002.

7. K. Beyls and E. D'Hollander. Generating cache hints for improved program efficiency. Journal of Systems Architecture, 51(4):223-250, 2005.

8. A. J. C. Bik. Compiler Support for Sparse Matrix Computations. $\mathrm{PhD}$ thesis, University of Leiden, The Netherlands, 1996.

9. B. Boigelot and L. Latour. Counting the solutions of Presburger equations without enumerating them. Theoretical Computer Science, 313(1):17-29, Feb. 2004.

10. P. Boulet and X. Redon. Communication pre-evaluation in HPF. In EUROPAR'98, volume 1470 of LNCS, pages 263-272. Springer Verlag, 1998.

11. V. Braberman, D. Garbervetsky, and S. Yovine. On synthesizing parametric specifications of dynamic memory utilization. Technical Report TR-2004-03, VERIMAG, Oct. 2003.

12. M. Brion. Points entiers dans les polyèdres convexes. Ann. Sci. École Norm. Sup. (4), 21(4):653-663, 1988.

13. S. Chatterjee, E. Parker, P. J. Hanlon, and A. R. Lebeck. Exact analysis of the cache behavior of nested loops. In Proceedings of the ACM SIGPLAN 2001 Conference on Programming Language Design and Implementation, pages 286-297. ACM Press, 2001.

14. P. Clauss and V. Loechner. Parametric analysis of polyhedral iteration spaces. Journal of VLSI Signal Processing, 19(2):179-194, July 1998.

15. P. D'Alberto, A. Veidembaum, A. Nicolau, and R. Gupta. Static analysis of parameterized loop nests for energy efficient use of data caches. In Workshop on Compilers and Operating Systems for Low Power (COLP01), Sept. 2001.

16. J. A. De Loera, D. Haws, R. Hemmecke, P. Huggins, B. Sturmfels, and R. Yoshida. Short rational functions for toric algebra and applications, 2004.

17. J. A. De Loera, D. Haws, R. Hemmecke, P. Huggins, J. Tauzer, and R. Yoshida. A user's guide for LattE v1.1, Nov. 2003. software package LattE is available at http: //www. math. ucdavis.edu/ latte/.

18. J. A. De Loera, R. Hemmecke, J. Tauzer, and R. Yoshida. Effective lattice point counting in rational convex polytopes. Journal of Symbolic Computation, 38(4):1273-1302, 2004.

19. S. Derrien, A. Turjan, C. Zissulescu, B. Kienhuis, and E. Deprettere. Deriving efficient control in Kahn process network. In Proc. of the "Int. Workshop on Systems, Architectures, Modeling, and Simulation, (SAMOS 2003)", 2003.

20. H. Edelsbrunner. Algorithms in combinatorial geometry. Springer-Verlag New York, Inc., 1987.

21. E. Ehrhart. Polynômes arithmétiques et méthode des polyèdres en combinatoire. International Series of Numerical Mathematics, 35, 1977.

22. P. Feautrier. Parametric integer programming. Operationnelle/Operations Research, 22(3):243-268, 1988.

23. J. Ferrante, V. Sarkar, and W. Thrash. On estimating and enhancing cache effectiveness. In U. Banerjee, D. Gelernter, A. Nicolau, and D. Padua, editors, Proceedings of the Fourth International Workshop on Languages and Compilers for Parallel Computing, volume 589 of Lecture Notes in Computer Science, pages 328-343. Springer-Verlag, Aug. 1991. 
24. D. Fimmel and R. Merker. Design of processor arrays for real-time applications. In Proc. Int. Conf. Euro-Par '98, Lecture Notes in Computer Science, pages 1018-1028, 1998.

25. B. Franke and M. O'Boyle. Array recovery and high-level transformations for DSP applications. ACM Transactions on Embedded Computing Systems, 2(2):132-162, May 2003.

26. Free Software Foundation, Inc. GMP. Available from ftp: / / ftp.gnu.org/gnu/gmp.

27. S. Ghosh, M. Martonosi, and S. Malik. Cache miss equations: a compiler framework for analyzing and tuning memory behavior. ACM Transactions on Programming Languages and Systems, 21(4):703-746, 1999.

28. N. Halbwachs, D. Merchat, and C. Parent-Vigouroux. Cartesian factoring of polyhedra in linear relation analysis. In Static Analysis Symposium, SAS'03, San Diego, June 2003. LNCS 2694, Springer Verlag.

29. F. Hannig and J. Teich. Design space exploration for massively parallel processor arrays. In Proceedings of the Sixth International Conference on Parallel Computing Technologies, volume 2127 of Lecture Notes in Computer Science, pages 51-65, 2001.

30. F. Heine and A. Slowik. Volume driven data distribution for NUMA-machines. In Proceedings from the 6th International Euro-Par Conference on Parallel Processing, pages 415-424, 2000.

31. P. Henrici. Applied and computational complex analysis. Wiley classics library. Wiley New York (N.Y.), 1974.

32. W. Kelly, V. Maslov, W. Pugh, E. Rosser, T. Shpeisman, and D. Wonnacott. The Omega calculator and library. Technical report, University of Maryland, Nov. 1996.

33. H. S. Kim, N. Vijaykrishnan, M. Kandemir, E. Brockmeyer, F. Catthoor, and M. J. Irwin. Estimating influence of data layout optimizations on SDRAM energy consumption. In Proceedings of the 2003 International Symposium on Low Power Electronics and Design, pages 40-43. ACM Press, 2003.

34. P. G. Kjeldsberg, F. Catthoor, and E. J. Aas. Data dependency size estimation for use in memory optimization. IEEE Transactions on Computer-Aided Design of Integrated Circuits and Systems, 22(7), July 2003.

35. B. Lisper. Fully automatic, parametric worst-case execution time analysis. In J. Gustafsson, editor, Proc. Third International Workshop on Worst-Case Execution Time (WCET) Analysis, pages 77-80, Porto, July 2003.

36. V. Loechner. Contribution à l'étude des polyèdres paramétrés et applications en parallélisation automatique. PhD thesis, University Louis Pasteur, Strasbourg, 1997.

37. V. Loechner. Polylib: A library for manipulating parameterized polyhedra. Technical report, ICPS, Université Louis Pasteur de Strasbourg, France, Mar. 1999.

38. V. Loechner, B. Meister, and P. Clauss. Precise data locality optimization of nested loops. J. Supercomput., 21(1):37-76, 2002.

39. V. Loechner and D. K. Wilde. Parameterized polyhedra and their vertices. International Journal of Parallel Programming, 25(6):525-549, Dec. 1997.

40. B. Nootaert, K. Beyls, and E. D'Hollander. On the calculation of Erhart polynomials in degenerate domains. Technical Report 105.001, Ghent University, Belgium, 2005.

41. E. Parker and S. Chatterjee. An automata-theoretic algorithm for counting solutions to Presburger formulas. In Compiler Construction 2004, volume 2985 of Lecture Notes in Computer Science, pages 104-119, Apr. 2004.

42. W. Pugh. Counting solutions to Presburger formulas: How and why. In SIGPLAN Conference on Programming Language Design and Implementation (PLDI'94), pages 121-134, 1994. 
43. A. Schrijver. Theory of Linear and Integer Programming. John Wiley \& Sons, 1986.

44. V. Shoup. NTL. Available from http://www. shoup. net/nt l/.

45. R. P. Stanley. Enumerative combinatorics, volume 1. Cambridge University Press, 1986.

46. B. Sturmfels. On vector partition functions. J. Comb. Theory Ser. A, 72(2):302-309, 1995.

47. E. Su, A. Lain, S. Ramaswamy, D. J. Palermo, E. W. Hodges IV, and P. Banerjee. Advanced compilation techniques in the PARADIGM compiler for distributed-memory multicomputers. In International Conference on Supercomputing, pages 424-433, 1995.

48. A. Turjan, B. Kienhuis, and E. Deprettere. A compile time based approach for solving out-of-order communication in Kahn process networks. In IEEE 13th International Conference on Application-specific Systems, Architectures and Processors (ASAP'2002), July 2002.

49. S. Verdoolaege, K. Beyls, M. Bruynooghe, and F. Catthoor. Experiences with enumeration of integer projections of parametric polytopes. In R. Bodik, editor, Proceedings of 14th International Conference on Compiler Construction, Edinburgh, Scotland, volume 3443 of Lecture Notes in Computer Science, pages 91-105, 2005.

50. S. Verdoolaege, R. Seghir, K. Beyls, V. Loechner, and M. Bruynooghe. Analytical computation of Ehrhart polynomials: Enabling more compiler analyses and optimizations. In Proceedings of International Conference on Compilers, Architectures, and Synthesis for Embedded Systems, Washington D.C., pages 248-258, Sept. 2004.

51. R. Yoshida. Barvinok's Rational Functions: Algorithms and Applications to Optimization, Statistics, and Algebra. PhD thesis, UC-Davis, 2004.

52. Y. Zhao and S. Malik. Exact memory size estimation for array computations. IEEE Transactions on Very Large Scale Integration (VLSI) Systems, 8(5):517-521, October 2000. 